\title{
SINGULAR LIMITS FOR 2-DIMENSIONAL ELLIPTIC PROBLEM WITH EXPONENTIALLY DOMINATED NONLINEARITY AND A QUADRATIC CONVECTION TERM
}

Sami Baraket, Imen Bazarbacha, Saber Kharrati and Taieb Ouni

\begin{abstract}
We study existence of solutions with singular limits for a two-dimensional semilinear elliptic problem with exponential dominated nonlinearity and a quadratic convection non linear gradient term, imposing Dirichlet boundary condition. This paper extends previous results obtained in $[1],[3],[4]$ and some references therein for related issues.
\end{abstract}

\section{Introduction and statement of the results}

In this work we are concerned with the following types of stationary singular problems:

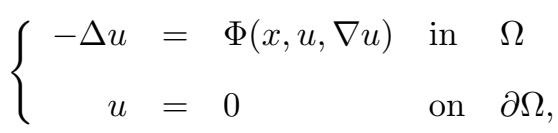

where $\Phi$ is a smooth nonlinear function and $\Omega$ is an open smooth bounded subset of $\mathbb{R}^{2}$. We focus on Problem (1) and we establish several recent contributions in the study of this equation. The most natural case where

\footnotetext{
Key Words: singular limits, Green's function, gradient term with quadratic growth, nonlinear domain decomposition method.

2010 Mathematics Subject Classification: 35J60, 53C21, 58J05.

Received: January, 2012

Accepted: February, 2012.
} 
$\Phi(x, u, \nabla u)=\lambda|\nabla u|^{2}+\varepsilon^{2} V(x)\left(e^{u}+e^{\gamma u}\right)$ where $\varepsilon$ and $\lambda$ are small parameters, $\gamma \in(0,1)$ and $V: \Omega \longrightarrow[0,+\infty)$ is a positive function. We deal with very general growth for $V(x)$ like, for example,

$$
V(x)=\prod_{i=1}^{N}\left|x-p_{i}\right|^{2 \beta_{i}} f(x),
$$

where $f$ is a smooth positive function such that $f\left(p_{i}\right)>0$, for $i=1, \cdots, N$ and $\left(\beta_{i}\right)_{1 \leq i \leq N}$ are positive numbers, so that problem (1) becomes

$$
\left\{\begin{array}{rll}
-\Delta u-\lambda|\nabla u|^{2} & =\varepsilon^{2} \prod_{i=1}^{N}\left|x-p_{i}\right|^{2 \beta_{i}} f(x)\left(e^{u}+e^{\gamma u}\right) & \text { in } \Omega \\
u=0 & \text { on } \partial \Omega .
\end{array}\right.
$$

Denote by $\Lambda:=\left\{p_{1}, \cdots, p_{N}\right\} \subset \Omega$ the set of singular sources. We are interested in solutions which "concentrate" as the parameters $\varepsilon$ and $\lambda$ tend to 0 (in a sense that will be determined later).

The aim of this paper is to study the influence of the non-linear gradient term $|\nabla u|^{2}$. It turns out that the presence of this term can have significant influence on the existence of a solution, as well as on its asymptotic behavior. The general question is: Does there exist a family of solutions $\left(u_{\varepsilon, \lambda, \beta_{i}}\right)_{\varepsilon>0, \lambda>0}$ of some singularly perturbed problem like (2) which converges to a non trivial singular function (on some set $\Lambda$ ) as the parameters $\varepsilon$ and $\lambda$ tend to 0 ?

Given $\bar{\beta}=\min _{1 \leq i \leq N} \beta_{i}>0$, for $\left(\beta_{i}\right)_{1 \leq i \leq N} \in \mathbb{R}_{+} \backslash \mathbb{N}$, we will suppose in the following that $\lambda$ satisfies

$$
\left(H_{\lambda}\right): \quad \lim _{\substack{\varepsilon \rightarrow 0 \\ \lambda \rightarrow 0}} \lambda \varepsilon^{-\delta /(\bar{\beta}+1)}=0, \quad \text { for any } \delta \in(0,1) .
$$

For $\beta=0$, we will suppose in the following that $\lambda$ satisfies

$$
\left(C_{\lambda}\right): \quad \text { If } 0<\varepsilon<\lambda \text {, then } \quad \lim _{\lambda \longrightarrow 0} \lambda^{1+\delta / 2} \varepsilon^{-\delta}=0, \quad \text { for any } \delta \in(0,1) \text {. }
$$

For $\lambda \equiv 0$, Esposito in [13] gave a positive answer to the above question for the following problem:

$$
\left\{\begin{aligned}
-\Delta u & =\varepsilon^{2} e^{u}-4 \pi \sum_{i=1}^{N} \beta_{i} \delta_{p_{i}} & & \text { in } \quad \\
u & =0 & & \text { on } \quad \partial \Omega
\end{aligned}\right.
$$

where again $\Omega \subset \mathbb{R}^{2}$ is an open smooth bounded subset of $\mathbb{R}^{2}, \delta_{p_{i}}$ are the Dirac mass at $p_{i}$ and $\Lambda:=\left\{p_{1}, \cdots, p_{N}\right\} \subset \Omega$ is the set of singular sources. 
Notice that using suitable transformation, problem (3), is equivalent to the following

$$
\left\{\begin{aligned}
-\Delta u & =\varepsilon^{2} \prod_{i=1}^{N}\left|x-p_{i}\right|^{2 \beta_{i}} f(x) e^{u} & \text { in } & \Omega \\
u & =0 & \text { on } & \partial \Omega,
\end{aligned}\right.
$$

where $f: \Omega \longrightarrow \mathbb{R}$ is a smooth function such that $f\left(p_{i}\right)>0$ for any $i=$ $1, \cdots, N$ and $\beta_{i}$ are positives numbers.

This type of equation has been studied by Bartolucci et al. in [8] and by Chen and Lin in [10]. They obtained the existence, sharp estimates and construction of multiple bubbles to (4). That the construction of nontrivial branches of solutions of such semilinear elliptic equations with exponential nonlinearities is equivalent to prove the existence of a conformal change of metric for which the corresponding mean curvature surfaces in the Euclidean space is non constant function.

Baraket et al. in [3] extended these results for special nonlinearities of type $e^{u}+e^{\gamma u}$ for $\gamma \in(0,1)$ instated of $e^{u}$. Problem $(4)_{\mid \beta_{i}=0}$ (a.e: $\Lambda$ is empty) is given by

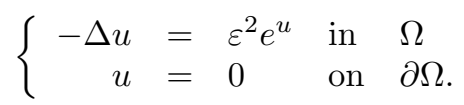

The above equation was first studied by Liouville in 1853, see [15], in which he derived a representation formula for all solutions of (5) which are defined in the hole space $\mathbb{R}^{2}$. When the parameter $\varepsilon$ tends to 0 , the asymptotic behavior of nontrivial branches of solutions of (5) is well understood thanks to the pioneer work of Suzuki [20] who characterizes the possible limit of nontrivial branches of solutions of (5). See also [18]. The existence of nontrivial branches of solutions was first proved by Weston [24] and then a general result has been obtained by Baraket and Pacard [6] and Baraket and Ye [7] for $e^{u}+e^{\gamma u}$, $\gamma \in(0,1)$ instated of $e^{u}$.These results were extended, applying to the ChernSimons vortex theory in mind, by Esposito et al. in [14] and Del Pino et al. in [12] to handle equations of the form

$$
-\Delta u=\varepsilon^{2} V e^{u}
$$

where $V$ is a non constant (positive) function and $\varepsilon$ is a small parameter. Let us mention that the construction of nontrivial branches of solutions of semilinear elliptic equations with exponential nonlinearities allowed Wente to provide counter examples to a conjecture by Hopf [23] concerning the existence of compact (immersed) constant mean curvature surfaces in the Euclidean space, see also [22] and [25] and some references therein.

To describe our result, we first introduce the Green's function $G\left(x, x^{\prime}\right)$ defined 
on $\Omega \times \Omega$, to be solution of

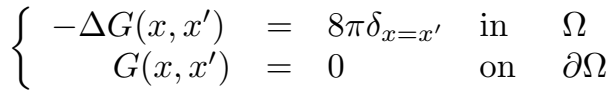

and let

$$
H\left(x, x^{\prime}\right)=G\left(x, x^{\prime}\right)+4 \log \left|x-x^{\prime}\right|,
$$

its regular part.

Given a finite subset $S=\left\{q_{1}, \cdots, q_{K}\right\}$ of $\Omega, m \in \mathbb{N}$ and $s \in\{1, \cdots, N\}$, we set

$$
\begin{aligned}
\mathcal{F}\left(x_{1}, \cdots, x_{m}\right) & =\sum_{j=1}^{m} H\left(x_{j}, x_{j}\right)+\sum_{i \neq j} G\left(x_{i}, x_{j}\right) \\
& +4 \sum_{i=1}^{s} \sum_{j=1}^{m} \beta_{i} \log \left(\left|x_{j}-p_{i}\right|\right)+2 \sum_{j=1}^{m} \log \left(f\left(x_{j}\right)\right)
\end{aligned}
$$

which is well defined in $(\Omega \backslash \Lambda)^{m}$ for any $x_{i} \neq x_{j}$ with $i \neq j$ and let

$$
\mathcal{G}\left(x_{1}, \cdots, x_{m}, w_{1}, \cdots, w_{s}\right)=\sum_{j=1}^{m} \sum_{i=1}^{s}\left(1+\beta_{i}\right) G\left(x_{j}, w_{i}\right) .
$$

$\mathcal{G}$ is well defined for $x_{j} \neq w_{i}$ with $x_{j} \in \Omega, w_{i} \in \Omega$.

The main result of this paper is the following.

Theorem 1. Given $\alpha \in(0,1)$. Let $\Omega$ be an open smooth bounded domain in $\mathbb{R}^{2}, f$ a smooth positive function and $\left(\beta_{i}\right)_{1 \leq i \leq n} \in \mathbb{R}_{+} \backslash \mathbb{N}^{*}$ be real numbers.

(a) Let $S=\left\{p_{j_{1}}, \cdots, p_{j_{s}}\right\} \subset \Lambda$, given $\lambda$ satisfying the condition $\left(H_{\lambda}\right)$, then there exist $\varepsilon_{0}>0, \lambda_{0}>0$, and $\left\{u_{\varepsilon, \lambda, \beta_{j_{i}}}\right\}_{\substack{0<\varepsilon<\varepsilon_{0} \\ 0<\lambda<\lambda_{0}}}$ of solutions of problem (2) such that

$$
\lim _{\substack{\varepsilon \rightarrow 0 \\ \lambda \rightarrow 0}} u_{\varepsilon, \lambda, \beta_{j_{i}}}=\sum_{i=1}^{s}\left(1+\beta_{j_{i}}\right) G\left(\cdot, p_{j_{i}}\right) \quad \text { in } \quad \mathcal{e}_{l o c}^{2, \alpha}(\Omega \backslash S) .
$$

(b) Let $S=\left\{q_{1}, \cdots, q_{m}\right\} \subset \Omega \backslash \Lambda$ and $\left(q_{1}, \cdots, q_{m}\right)$ be a nondegenerate critical point of the functional $\mathcal{F}$, given $\lambda$ satisfying the condition $\left(C_{\lambda}\right)$, then there exist $\varepsilon_{0}>0, \lambda_{0}>0$, and $\left\{u_{\varepsilon, \lambda}:=\left(u_{\varepsilon, \lambda, 0}\right)\right\}_{\substack{0<\varepsilon<\varepsilon_{0} \\ 0<\lambda<\lambda_{0}}}$ of solutions of problem (2) such that

$$
\lim _{\substack{\varepsilon \rightarrow 0 \\ \lambda \rightarrow 0}} u_{\varepsilon, \lambda}=\sum_{i=1}^{m} G\left(\cdot, q_{i}\right) \quad \text { in } \quad \mathcal{C}_{l o c}^{2, \alpha}(\Omega \backslash S) .
$$


(c) Let $S$ be such that $S \cap \Lambda=\left\{p_{j_{1}}, \cdots, p_{j_{s}}\right\}, S \backslash \Lambda=\left\{q_{1}, \cdots, q_{m}\right\}$ and $\left(q_{1}, \cdots, q_{m}\right)$ be a nondegenerate critical point of the function $\mathcal{F}(\cdot)+$ $\mathcal{G}\left(\cdot, p_{j_{1}}, \cdots, p_{j_{s}}\right)$, given $\lambda$ satisfying the conditions $\left(H_{\lambda}\right)$ and $\left(C_{\lambda}\right)$, then there exist $\varepsilon_{0}>0, \lambda_{0}>0$, and $\left\{u_{\varepsilon, \lambda, \beta_{j_{i}}}\right\}_{\substack{0<\varepsilon<\varepsilon_{0} \\ 0<\lambda<\lambda_{0}}}$ of solutions of (2) such that

$$
\lim _{\substack{\varepsilon \rightarrow 0 \\ \lambda \rightarrow 0}} u_{\varepsilon, \lambda, \beta_{j_{i}}}=\sum_{k=1}^{s}\left(1+\beta_{j_{k}}\right) G\left(\cdot, p_{j_{k}}\right)+\sum_{i=1}^{m} G\left(\cdot, q_{i}\right) \quad \text { in } \quad \mathcal{C}_{l o c}^{2, \alpha}(\Omega \backslash S) .
$$

In the case where $\Lambda$ is empty and taking $f$ a positive constant funcion, part (b) of Theorem 1 extends the result in [1].

Observe that the nondegeneracy assumption on critical points, if it exists, is a rather mild assumption since it is certainly fulfilled for a generic choice of the domain $\Omega$.

\section{Construction of the approximate solution}

We first describe the rotationally symmetric approximate solutions of

$$
\Delta v+\lambda|\nabla v|^{2}+\varepsilon^{2}|x|^{2 \beta}\left(e^{v}+e^{\gamma v}\right)=0
$$

in $\mathbb{R}^{2}$ where $\beta \in \mathbb{R}_{+} \backslash \mathbb{N}$ and $\gamma \in(0,1)$.

We define the function

$$
v_{\varepsilon, \beta}(x):=\log 8(\beta+1)^{2}-2 \log \left(\varepsilon^{2}+|x|^{2(1+\beta)}\right)
$$

solution of the equation

$$
-\Delta v=\varepsilon^{2}|x|^{2 \beta} e^{v} \quad \text { in } \mathbb{R}^{2} .
$$

Notice that equation (10) is invariant under dilation in the following sense : If $v$ is a solution of (10) and if $\tau>0$, then $v(\tau \cdot)+2(\beta+1) \log \tau$ is also a solution of (10). With this observation in mind, we define for all $\tau>0$

$$
v_{\varepsilon, \tau, \beta}(x):=2(\beta+1) \log \tau+\log 8(\beta+1)^{2}-2 \log \left(\varepsilon^{2}+|\tau x|^{2(1+\beta)}\right)
$$

and

$$
v_{\varepsilon, \tau}(x):=v_{\varepsilon, \tau, 0}(x) .
$$




\subsection{A linearized operator on $B_{1}$}

We set $B_{1}^{*}=B_{1}-\{0\}$. Then we introduce the following space:

Definition 1. Given $k \in \mathbb{N}, \alpha \in(0,1)$ and $\mu \in \mathbb{R}$, we introduce the Hölder weighted spaces $\mathrm{C}_{\mu}^{k, \alpha}\left(\bar{B}_{1}^{*}\right)$ as the space of functions in $\mathrm{C}_{\text {loc }}^{k, \alpha}\left(\bar{B}_{1}^{*}\right)$ for which the following norm

$$
\|u\|_{\mathcal{C}_{\mu}^{k, \alpha}\left(\bar{B}_{1}^{*}\right)}=\sup _{r \leq 1 / 2}\left(r^{-\mu}\|u(r \cdot)\|_{\mathfrak{C}^{k, \alpha}\left(\bar{B}_{2}-B_{1}\right)}\right),
$$

is finite.

We then define the subspace of radial functions in $\mathcal{C}_{\mu}^{k, \alpha}\left(\bar{B}_{1}^{*}\right)$ by

$$
\mathcal{C}_{\text {rad, }, \mu}^{k, \alpha}\left(\bar{B}_{1}^{*}\right)=\left\{f \in \mathcal{C}_{\mu}^{k, \alpha}\left(\bar{B}_{1}^{*}\right) ; \text { such that } f(x)=f(|x|), \forall x \in \bar{B}_{1}^{*}\right\} .
$$

We define the linear second order elliptic operator $\mathbb{L}_{\beta}$ by

$$
\mathbb{L}_{\beta}:=\Delta+\varepsilon^{2}|x|^{2 \beta} e^{v_{\varepsilon, \tau, \beta}}
$$

which corresponds to the linearization of $\Delta u+\varepsilon^{2}|x|^{2 \beta} e^{u}=0$ about the radial symmetric solutions $v_{\varepsilon, \tau, \beta}$ for both cases $\beta=0$ and $\beta \notin \mathbb{N}$.

When $k \geq 2$, we let $\left[\mathrm{C}_{\mu}^{k, \alpha}(\bar{\Omega})\right]_{0}$ be the subspace of functions $w \in \mathcal{C}_{\mu}^{k, \alpha}(\bar{\Omega})$ satisfying $w=0$ on $\partial \bar{\Omega}$. We recall the result in [13] which states as:

Proposition 1. [6],[13]

(i) Let $\mu>0, \mu \notin \mathbb{N}$ and $\beta \notin \mathbb{N}$, then $\mathbb{L}_{\beta}:\left[\mathcal{C}_{\mu}^{2, \alpha}\left(B_{1}\right)\right]_{0} \longrightarrow \mathcal{C}_{\mu-2}^{0, \alpha}\left(B_{1}\right)$ is surjective.

(ii) Let $\mu>0, \mu \notin \mathbb{N}$ and $\beta=0$, then $\mathbb{L}_{0}:\left[\mathcal{C}_{\text {rad, }, \mu}^{2, \alpha}\left(B_{1}\right)\right]_{0} \longrightarrow \mathcal{C}_{\text {rad, }, \mu-2}^{0, \alpha}\left(B_{1}\right)$ is surjective.

(iii) Let $\mu>1, \mu \notin \mathbb{N}$ and $\beta=0$, then $\mathbb{L}_{0}:\left[\mathcal{C}_{\mu}^{2, \alpha}\left(B_{1}\right)\right]_{0} \longrightarrow \mathcal{C}_{\mu-2}^{0, \alpha}\left(B_{1}\right)$ is surjective.

In the following, we denote by $\mathcal{G}_{\mu, \beta}$ a right inverse of $\mathbb{L}_{\beta}$. Let

$$
\delta \in(0,1),
$$

we define

$$
r_{\varepsilon, \beta}:=\max \left(\varepsilon^{2(1-\gamma)-\delta / 2(\beta+1)}, \lambda^{1 / 2} \varepsilon^{-\delta / 2(\beta+1)}, \lambda^{1 / 2}, \varepsilon^{2 /(2 \beta+3)}\right)
$$

and

$$
r_{\varepsilon}:=\max \left(\varepsilon^{2(1-\gamma)-\delta / 2}, \lambda^{1 / 2} \varepsilon^{-\delta / 2}, \lambda^{1 / 2}, \varepsilon^{1 / 2}\right) .
$$


Remark that by assumptions $\left(H_{\lambda}\right)$ and $\left(C_{\lambda}\right), r_{\varepsilon, \beta}$ and $r_{\varepsilon}$ tend to 0 respectively as $\varepsilon$ and $\lambda$ tend to 0 .

We would like to find a solution $v$ of

$$
\Delta v+\lambda|\nabla v|^{2}+\varepsilon^{2}|x|^{2 \beta}\left(e^{v}+e^{\gamma v}\right)=0
$$

in $B_{r_{\varepsilon, \beta}}$ of the form

$$
v(x)=v_{\varepsilon, \tau, \beta}(x)+h(x) .
$$

Equation (13) yields

$$
\begin{aligned}
\mathbb{L}_{\beta} h & =-\frac{8(\beta+1)^{2} \tau^{2(\beta+1)} \varepsilon^{2}|x|^{2 \beta}}{\left(\varepsilon^{2}+|\tau x|^{2(\beta+1)}\right)^{2}}\left(e^{h}-h-1\right) \\
& -\frac{8^{\gamma}(\beta+1)^{2 \gamma} \tau^{2 \gamma(\beta+1)} \varepsilon^{2}|x|^{2 \beta}}{\left(\varepsilon^{2}+|\tau x|^{2(\beta+1)}\right)^{2 \gamma}} e^{\gamma h}-\lambda\left|\nabla\left(v_{\varepsilon, \tau, \beta}+h\right)\right|^{2}
\end{aligned}
$$

in $B_{r_{\varepsilon, \beta}}$.

By Proposition 1, solving the equation (14) is equivalent to find a fixed point $h$, in a small ball of $\mathcal{C}_{\delta}^{2, \alpha}\left(B_{r_{\varepsilon, \beta}}\right)$, solution of

$$
h=\mathcal{G}_{\delta, \beta} \circ \mathfrak{R}(h)
$$

where

$$
\begin{aligned}
\mathfrak{R}(h) & =-\frac{8(\beta+1)^{2} \tau^{2(\beta+1)} \varepsilon^{2}|x|^{2 \beta}}{\left(\varepsilon^{2}+|\tau x|^{2(\beta+1)}\right)^{2}}\left(e^{h}-h-1\right) \\
& -\frac{8^{\gamma}(\beta+1)^{2 \gamma} \tau^{2 \gamma(\beta+1)} \varepsilon^{2}|x|^{2 \beta}}{\left(\varepsilon^{2}+|\tau x|^{2(\beta+1)}\right)^{2 \gamma}} e^{\gamma h}-\lambda\left|\nabla\left(v_{\varepsilon, \tau, \beta}+h\right)\right|^{2} .
\end{aligned}
$$

We have

$$
|\Re(0)| \leq c_{\kappa} \varepsilon^{2}|x|^{2 \beta}\left(\varepsilon^{2}+|\tau x|^{2(\beta+1)}\right)^{-2 \gamma}+c_{\kappa} \lambda\left|\nabla v_{\varepsilon, \tau, \beta}\right|^{2},
$$

this implies that for $\gamma \in(0,1)$ and $|x|=r$ we have

$$
\begin{aligned}
\sup _{r \leq r_{\varepsilon, \beta}} r^{2-\delta}|\mathfrak{R}(0)| & \leq c_{\kappa} \varepsilon^{2} \sup _{r \leq r_{\varepsilon, \beta}}|x|^{2 \beta+2-\delta}\left(\varepsilon^{2}+|\tau x|^{2(\beta+1)}\right)^{-2 \gamma} \\
& +c_{\kappa} \sup _{r \leq r_{\varepsilon, \beta}} \lambda|x|^{4 \beta+4-\delta}\left(\varepsilon^{2}+|\tau x|^{2(\beta+1)}\right)^{-2} \\
& \leq c_{\kappa} \varepsilon^{4(1-\gamma)-\delta /(\beta+1)}+c_{\kappa} \lambda \varepsilon^{-\delta /(\beta+1)} \leq c_{\kappa} r_{\varepsilon, \beta}^{2}
\end{aligned}
$$


For $h_{1}, h_{2}$ in $B\left(0,2 c_{\kappa} r_{\varepsilon, \beta}^{2}\right)$ of $\mathcal{C}_{r a d, \delta}^{2, \alpha}\left(B_{r_{\varepsilon, \beta}}\right)$, we have for $\gamma \in(0,1)$, that

$$
\begin{aligned}
& \sup _{r \leq r_{\varepsilon, \beta}} r^{2-\delta}\left|\mathfrak{R}\left(h_{2}\right)-\mathfrak{R}\left(h_{1}\right)\right| \\
& \leq c_{\kappa} \varepsilon^{2} \sup _{r \leq r_{\varepsilon, \beta}} r^{2 \beta+2-\delta}\left(\varepsilon^{2}+|\tau x|^{2(\beta+1)}\right)^{-2}\left|e^{h_{2}}-e^{h_{1}}+h_{1}-h_{2}\right| \\
& +c_{\kappa} \varepsilon^{2} \sup _{r \leq r_{\varepsilon, \beta}} r^{2 \beta+2-\delta}\left(\varepsilon^{2}+|\tau x|^{2(\beta+1)}\right)^{-2 \gamma}\left|e^{\gamma h_{1}}-e^{\gamma h_{2}}\right| \\
& +c_{\kappa} \sup r^{2-\delta} \lambda\left(\left|\nabla\left(v_{\varepsilon, \tau, \beta}+h_{2}\right)\right|^{2}-\left|\nabla\left(v_{\varepsilon, \tau, \alpha}+h_{1}\right)\right|^{2}\right) \\
& \leq c_{\kappa} \varepsilon^{\delta /(\beta+1)} r_{\varepsilon, \beta}^{2}\left\|h_{2}-h_{1}\right\|_{\mathcal{C}_{\delta}^{2, \alpha}\left(B_{r_{\varepsilon, \beta}}\right)} \\
& +c_{\kappa} \varepsilon^{4(1-\gamma)-\delta /(\beta+1)}\left\|h_{2}-h_{1}\right\|_{\mathcal{C}_{\delta}^{2, \beta}\left(B_{r_{\varepsilon, \beta}}\right)}+c_{\kappa} \lambda\left\|h_{2}-h_{1}\right\|_{\mathcal{C}_{\delta}^{2, \beta}\left(B_{r_{\varepsilon, \beta}}\right)} \\
& \leq c_{\kappa} r_{\varepsilon, \beta}^{2}\left\|h_{2}-h_{1}\right\|_{\mathcal{C}_{\delta}^{2, \alpha}\left(B_{r_{\varepsilon, \beta}}\right)} \text {. }
\end{aligned}
$$

Thus, applying a classical fixed point argument, when $\varepsilon$ is small enough, we prove the existence and uniqueness of $h$, solution of (15). We summarize this in the following result.

Proposition 2. $i)$ Given $\delta \in(0,1-\gamma], \beta \notin \mathbb{N}, \lambda$ satisfying $\left(H_{\lambda}\right)$ and $\kappa>1$, then there exist $c_{\kappa}>0$ independent of $\varepsilon$ and a solution $h \in \mathcal{C}_{\text {rad, }, \delta}^{2, \alpha}\left(B_{r_{\varepsilon, \beta}}\right)$ of (15) satisfying

$$
\|h\|_{\mathcal{C}_{\delta}^{2, \alpha}\left(B_{r_{\varepsilon, \beta}}\right)} \leq 2 c_{\kappa} r_{\varepsilon, \beta}^{2}
$$

Moreover $u_{\varepsilon, \tau, \beta}(x)=v_{\varepsilon, \tau, \beta}(x)+h(x)$ is a solution of (13) in $B_{r_{\varepsilon, \beta}}$.

ii) Given $\delta \in(0,1), \beta=0, \lambda$ satisfying $\left(C_{\lambda}\right)$ and $\kappa>1$, then there exist $c_{\kappa}>0$ independent of $\varepsilon$ and $h \in \mathcal{C}_{\text {rad, } \delta}^{2, \alpha}\left(B_{r_{\varepsilon}}\right)$ solution of $(15)_{\mid \beta=0}$ satisfying

$$
\|h\|_{\mathcal{C}_{\delta}^{2, \alpha}\left(B_{r_{\varepsilon}}\right)} \leq 2 c_{\kappa} r_{\varepsilon}^{2}
$$

Moreover $u_{\varepsilon, \tau}(x)=v_{\varepsilon, \tau}(x)+h(x)$ is a solution of $(13)_{\mid \beta=0}$ in $B_{r_{\varepsilon}}$.

\subsection{Analysis of the Laplace operator in weighted spaces [4]}

In this section, we study the mapping properties of the Laplace operator in weighted Hölder spaces. Given $q_{1}, \ldots, q_{K} \in \Omega$, we set $\mathbf{q}:=\left(q_{1}, \cdots, q_{K}\right)$ and

$$
\bar{\Omega}^{*}(\mathbf{q}):=\bar{\Omega}-\left\{q_{1}, \ldots q_{K}\right\},
$$

and we choose $r_{0}>0$ so that the balls $B_{r_{0}}\left(q_{j}\right)$ of center $q_{j}$ and radius $r_{0}$ are mutually disjoint and included in $\Omega$. For all $r \in\left(0, r_{0}\right)$, we define

$$
\bar{\Omega}_{r}(\mathbf{q}):=\bar{\Omega}-\bigcup_{j=1}^{K} B_{r}\left(q_{j}\right) .
$$


Definition 2. Given $k \in \mathbb{R}, \alpha \in(0,1)$ and $\nu \in \mathbb{R}$, we introduce the Hölder weighted space $\mathrm{C}_{\nu}^{k, \alpha}\left(\bar{\Omega}^{*}(\mathbf{q})\right)$ as the space of functions $w \in \mathrm{C}_{\text {loc }}^{k, \alpha}\left(\bar{\Omega}^{*}(\mathbf{q})\right)$ for with the following norm

$$
\begin{aligned}
\|w\|_{\mathcal{C}_{\nu}^{k, \alpha}\left(\bar{\Omega}^{*}(\mathbf{q})\right)}: & =\|w\|_{\mathfrak{C}^{k, \alpha}\left(\bar{\Omega}-\cup_{j=1}^{K} B_{r_{0} / 2}\left(q_{j}\right)\right)} \\
& +\sum_{j=1}^{K} \sup _{0<r \leq r_{0} / 2} r^{-\nu}\left\|w\left(q_{j}+r \cdot\right)\right\|_{\mathfrak{C}^{k, \alpha}\left(\bar{B}_{2}-B_{1}\right)}
\end{aligned}
$$

is finite.

When $k \geq 2$, we denote by $\left[\mathcal{C}_{\nu}^{k, \alpha}\left(\bar{\Omega}^{*}(\mathbf{q})\right)\right]_{\mathbf{0}}$ the subspace of functions $w \in$ $\mathcal{C}_{\nu}^{k, \alpha}\left(\bar{\Omega}^{*}(\mathbf{q})\right)$ satisfying $w=0$ on $\partial \Omega$.

Proposition 3. Assume that $\nu<0$ and $\nu \notin \mathbb{Z}$, then

$$
\begin{array}{cccc}
\mathcal{L}_{\nu}:\left[\mathcal{C}_{\nu}^{2, \alpha}\left(\bar{\Omega}^{*}(\mathbf{q})\right)\right]_{\mathbf{0}} & \longrightarrow & \mathcal{C}_{\nu-2}^{0, \alpha}\left(\bar{\Omega}^{*}(\mathbf{q})\right) \\
w & \longmapsto & \Delta w
\end{array}
$$

is surjective. .

In the following, we denote by $\tilde{\mathcal{G}}_{\nu}$ a right inverse of $\mathcal{L}_{\nu}$.

Remark 1. Observe that, when $\nu<0, \nu \notin \mathbb{Z}$, a right inverse is not unique and depends smoothly on the points $q_{1}, \ldots, q_{m}$, at least locally. Once a right inverse is fixed for one choice of the points $q_{1}, \ldots, q_{K}$, a right inverse for another choice of points $\tilde{q}_{1}, \ldots, \tilde{q}_{K}$ close to $q_{1}, \ldots, q_{K}$ can be obtained by using a simple perturbation argument.

\subsection{Harmonic extensions [4]}

We study the properties of interior and exterior harmonic extensions. Given $\varphi \in \mathcal{C}^{2, \alpha}\left(S^{1}\right)$, we define $H^{i}\left(=H^{i}(\varphi ; \cdot)\right)$ to be the solutions of

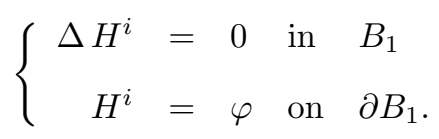

We denote by $e_{1}, e_{2}$ the coordinate functions on $S^{1}$.

Lemma 1. i) If we assume that

$$
\int_{S^{1}} \varphi d v_{S^{1}}=0
$$


then there exists $c>0$ such that

$$
\left\|H^{i}(\varphi ; \cdot)\right\|_{\mathfrak{C}_{1}^{2, \alpha}\left(\bar{B}_{1}^{*}\right)} \leq c\|\varphi\|_{\mathfrak{C}^{2, \alpha}\left(S^{1}\right)} .
$$

ii) If we assume that

$$
\int_{S^{1}} \varphi d v_{S^{1}}=0 \quad \text { and } \quad \int_{S^{1}} \varphi e_{\ell} d v_{S^{1}}=0 \quad \text { for } \quad \ell=1,2
$$

then there exists $c>0$ such that

$$
\left\|H^{i}(\varphi ; \cdot)\right\|_{\mathcal{C}_{2}^{2, \alpha}\left(\bar{B}_{1}^{*}\right)} \leq c\|\varphi\|_{\mathcal{C}^{2, \alpha}\left(S^{1}\right)} .
$$

Given $\varphi \in \mathcal{C}^{2, \alpha}\left(S^{1}\right)$ we define $H^{e}\left(=H^{e}(\varphi ; \cdot)\right)$ to be the solution of

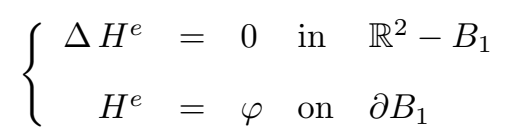

which decays at infinity.

Definition 3. Given $k \in \mathbb{N}, \alpha \in(0,1)$ and $\nu \in \mathbb{R}$, we define the space $\mathcal{C}_{\nu}^{k, \alpha}\left(\mathbb{R}^{2}-B_{1}\right)$ as the space of functions $w \in \mathcal{C}_{l o c}^{k, \alpha}\left(\mathbb{R}^{2}-B_{1}\right)$ for which the following norm

$$
\|w\|_{\mathcal{C}_{\nu}^{k, \alpha}\left(\mathbb{R}^{2}-B_{1}\right)}=\sup _{r \geq 1}\left(r^{-\nu}\|w(r \cdot)\|_{\mathcal{C}_{\nu}^{k, \alpha}\left(\bar{B}_{2}-B_{1}\right)}\right),
$$

is finite.

Lemma 2. If we assume that

$$
\int_{S^{1}} \varphi d v_{S^{1}}=0
$$

Then there exists $c>0$ such that

$$
\left\|H^{e}(\varphi, ; \cdot)\right\|_{\mathcal{C}_{-1}^{2, \alpha}\left(\mathbb{R}^{2}-B_{1}\right)} \leq c\|\varphi\|_{\mathcal{C}^{2, \alpha}\left(S^{1}\right)} .
$$

If $F \subset L^{2}\left(S^{1}\right)$ is a space of functions defined on $S^{1}$, we define the space $F^{\perp}$ to be the subspace of functions of $F$ which are $L^{2}\left(S^{1}\right)$-orthogonal to the functions $e_{1}, e_{2}$.

Lemma 3. The mapping

$$
\begin{aligned}
\mathcal{P}: \mathcal{C}^{2, \alpha}\left(S^{1}\right)^{\perp} & \longrightarrow \mathcal{C}^{1, \alpha}\left(S^{1}\right)^{\perp} \\
\varphi & \longmapsto \partial_{r} H^{i}-\partial_{r} H^{e}
\end{aligned}
$$

where $H^{i}=H^{i}(\varphi ; \cdot)$ and $H^{e}=H^{e}(\varphi ; \cdot)$, is an isomorphism. 


\section{The nonlinear interior problem}

We are interested in equations of type

$$
\Delta w+\lambda|\nabla w|^{2}+\varepsilon^{2} V(x)\left(e^{w}+e^{\gamma w}\right)=0 .
$$

First, we will treat the case where 0 is a zero of the potential $V$. Then, we can write $V(x)=|x|^{2 \beta} K(x)$ where $K$ is some smooth function such that $K(0)>0$. We would like to find $w$ solution of

$$
\Delta w+\lambda|\nabla w|^{2}+\varepsilon^{2}|x|^{2 \beta} K(x)\left(e^{w}+e^{\gamma w}\right)=0
$$

in $B_{r_{\varepsilon, \beta}}$. Given $\varphi \in \mathfrak{C}^{2, \alpha}\left(S^{1}\right)$ satisfying (20) and (21), we define

$$
\mathbf{v}:=u_{\varepsilon, \tau, \beta}-\log (K(0))+H^{i}\left(\varphi, \cdot / r_{\varepsilon, \beta}\right)
$$

then we look for a solution of (25) of the form $w=\mathbf{v}+v$ and using the fact that $H_{\beta}^{i}$ is harmonic, this amounts to solve

$$
-\mathbb{L}_{\beta} v=S(v)
$$

where

$$
\begin{aligned}
& S(v)=\frac{8(\beta+1)^{2} \tau^{2(\beta+1)} \varepsilon^{2}|x|^{2 \beta}}{\left(\varepsilon^{2}+|\tau x|^{2(1+\beta)}\right)^{2}} e^{H^{i}\left(\varphi, \cdot / r_{\varepsilon, \beta}\right)+h+v}\left(\frac{K(x)}{K(0)}-1\right) \\
&+\frac{8(\beta+1)^{2} \tau^{2(\beta+1)} \varepsilon^{2}|x|^{2 \beta}}{\left(\varepsilon^{2}+|\tau x|^{2(1+\beta)}\right)^{2}} e^{h}\left(e^{H^{i}\left(\varphi, \cdot / r_{\varepsilon, \beta}\right)+v}-v-1\right) \\
&+ \frac{8(\beta+1)^{2} \tau^{2(\beta+1)} \varepsilon^{2}|x|^{2 \beta}}{\left(\varepsilon^{2}+|\tau x|^{2(1+\beta)}\right)^{2}}\left(e^{h}-1\right) v \\
&+ \frac{8^{\gamma}(\beta+1)^{2 \gamma} \tau^{2 \gamma(\beta+1)} \varepsilon^{2}|x|^{2 \beta}}{\left(\varepsilon^{2}+|\tau x|^{2(\beta+1)}\right)^{2 \gamma}} e^{\gamma h}\left(\frac{K(x)}{K(0)^{\gamma}} e^{\gamma H^{i}\left(\varphi, \cdot / r_{\varepsilon, \beta}\right)+\gamma v}-1\right) \\
&+\lambda\left|\nabla\left[v_{\varepsilon, \tau, \beta}(x)+H^{i}\left(\varphi, x / r_{\varepsilon, \beta}\right)+h(x)+v(x)\right]\right|^{2} \\
&-\lambda\left|\nabla\left(v_{\varepsilon, \tau, \beta}(x)+h(x)\right)\right|^{2} .
\end{aligned}
$$

By Proposition 1, it is sufficient to find $v \in \mathcal{C}_{\mu}^{2, \alpha}\left(B_{r_{\varepsilon, \beta}}\right)$ solution of

$$
v=-\mathcal{G}_{\mu, \beta} \circ S(v)
$$


in $B_{r_{\varepsilon, \beta}}$. We denote by $\mathcal{N}\left(=\mathcal{N}_{\varepsilon, \tau, \beta, \varphi}\right)$ the nonlinear operator appearing on the right hand side of the above equation. Given $\kappa>0$ (whose value will be fixed later on), we further assume that the functions $\varphi$ satisfy

$$
\|\varphi\|_{\mathcal{C}^{2, \alpha}} \leq \kappa r_{\varepsilon, \beta} \quad \text { for } \beta \notin \mathbb{N}
$$

and

$$
\|\varphi\|_{\mathcal{C}^{2, \alpha}} \leq \kappa r_{\varepsilon}^{2} \quad \text { for } \beta=0 .
$$

Then, we have the following result.

Lemma 4. i) Let $\beta \notin \mathbb{N}$, under the above assumptions, there exists a constant $c_{\kappa}>0$ such that for $\mu \in(0,1-\gamma]$

$$
\|\mathcal{N}(0)\|_{\mathcal{C}_{\mu}^{2, \alpha}\left(B_{r_{\varepsilon, \beta}}\right)} \leq c_{\kappa} r_{\varepsilon, \beta}
$$

and

$$
\left\|\mathcal{N}\left(v_{2}\right)-\mathcal{N}\left(v_{1}\right)\right\|_{\mathcal{C}_{\mu}^{2, \alpha}\left(B_{r_{\varepsilon, \beta}}\right)} \leq c_{\kappa} r_{\varepsilon, \beta}\left\|v_{2}-v_{1}\right\|_{\mathcal{C}_{\mu}^{2, \alpha}\left(B_{r_{\varepsilon, \beta}}\right)}
$$

provided $v_{1}, v_{2} \in \mathcal{C}_{\mu}^{2, \alpha}\left(B_{r_{\varepsilon, \beta}}\right)$ satisfy $\left\|v_{i}\right\|_{\mathcal{C}_{\mu}^{2, \alpha}\left(B_{r_{\varepsilon, \beta}}\right)} \leq 2 c_{\kappa} r_{\varepsilon, \beta}$.

ii) Let $\beta=0$, under the above assumptions, there exists a constant $c_{\kappa}>0$ such that for $\mu \in(1,2)$

$$
\|\mathcal{N}(0)\|_{\mathcal{C}_{\mu}^{2, \alpha}\left(B_{r_{\varepsilon}}\right)} \leq c_{\kappa} r_{\varepsilon}^{2}
$$

and

$$
\left\|\mathcal{N}\left(v_{2}\right)-\mathcal{N}\left(v_{1}\right)\right\|_{\mathcal{C}_{\mu}^{2, \alpha}\left(B_{r_{\varepsilon}}\right)} \leq c_{\kappa} r_{\varepsilon}^{2}\left\|v_{2}-v_{1}\right\|_{\mathcal{C}_{\mu}^{2, \alpha}\left(B_{r_{\varepsilon}}\right)}
$$

provided $v_{1}, v_{2} \in \mathcal{C}_{\mu}^{2, \alpha}\left(B_{r_{\varepsilon}}\right)$ satisfying $\left\|v_{i}\right\|_{\mathcal{C}_{\mu}^{2, \alpha}\left(B_{r_{\varepsilon}}\right)} \leq 2 c_{\kappa} r_{\varepsilon}^{2}$.

Proof . The proof of the first estimate of part $i$ ) follows from the result of Lemma 1 part $i$ ) together with the assumption on the norms of $\varphi$. We briefly comment on how these are used: it follows from Lemma 1, part $i$ ) that for $\beta \notin \mathbb{N}$, we have

$$
\left\|H^{i}\left(\varphi, \cdot / r_{\varepsilon, \beta}\right)\right\|_{\mathcal{C}^{2, \alpha}\left(\bar{B}_{r_{\varepsilon, \beta}}\right)} \leq c_{\kappa} r r_{\varepsilon, \beta}^{-1}\|\varphi\|_{\mathcal{C}^{2, \alpha}\left(S^{1}\right)}
$$

for all $r \leq r_{\varepsilon, \beta} / 2$. Then using (27), we get

$$
\left\|H^{i}\left(\varphi, \cdot / r_{\varepsilon, \beta}\right)\right\|_{\mathcal{C}_{1}^{2, \alpha}\left(\bar{B}_{r_{\varepsilon, \beta}}\right)} \leq c_{\kappa} r_{\varepsilon, \beta}
$$

for all $r \leq r_{\varepsilon, \beta} / 2$.

Using the fact that $\|h\| \leq 2 c_{\kappa} r_{\varepsilon, \beta}^{2}$ which tends to 0 as $\varepsilon$ tends to 0 , then for $\mu \in(0,1-\gamma]$, we get

$$
\begin{aligned}
& \left\|\varepsilon^{2}|x|^{2 \beta}\left(\varepsilon^{2}+|\tau x|^{2(\beta+1)}\right)^{-2} e^{h}\left(e^{H^{i}\left(\varphi, \cdot / r_{\varepsilon, \beta}\right)}-1\right)\right\|_{\mathcal{C}_{\mu-2}^{0, \alpha}\left(\bar{B}_{r_{\varepsilon, \beta}}\right)} \\
& \leq c_{\kappa} \varepsilon^{(1-\mu) /(\beta+1)} \leq c_{\kappa} r_{\varepsilon, \beta},
\end{aligned}
$$




$$
\begin{aligned}
& \left\|\varepsilon^{2}|x|^{2 \beta}\left(\varepsilon^{2}+|\tau x|^{2(\beta+1)}\right)^{-2} e^{H^{i}\left(\varphi, \cdot / r_{\varepsilon, \beta}\right)+h}\left(\frac{K(x)}{K(0)}-1\right)\right\|_{\mathcal{C}_{\mu-2}^{0, \alpha}\left(\bar{B}_{r_{\varepsilon, \beta}}\right)} \\
& \leq c_{\kappa} \varepsilon^{(1-\mu) /(\beta+1)} \leq c_{\kappa} r_{\varepsilon, \beta}
\end{aligned}
$$

and

$$
\begin{aligned}
& \left\|\varepsilon^{2}|x|^{2 \beta}\left(\varepsilon^{2}+|\tau x|^{2(\beta+1)}\right)^{-2 \gamma} e^{\gamma h}\left(\frac{K(x)}{K(0)^{\gamma}} e^{\gamma H^{i}\left(\varphi, \cdot / r_{\varepsilon, \beta}\right)}-1\right)\right\|_{\mathcal{C}_{\mu-2}^{0, \beta}\left(\bar{B}_{r_{\varepsilon, \beta}}\right)} \\
& \leq c_{\kappa} \varepsilon^{4(1-\gamma)-\mu /(\beta+1)}\left(1+\varepsilon^{1 /(\beta+1)}\right) \leq c_{\kappa} r_{\varepsilon, \beta} .
\end{aligned}
$$

on the other hand, using the condition $\left(H_{\lambda}\right)$, we get

$$
\begin{aligned}
& \left\|\lambda\left|\nabla\left[v_{\varepsilon, \tau, \beta}(x)+H^{i}\left(\varphi, x / r_{\varepsilon, \beta}\right)+h(x)\right]\right|^{2}-\lambda\left|\nabla\left[v_{\varepsilon, \tau, \beta}(x)+h(x)\right]\right|^{2}\right\|_{\mathcal{C}_{\mu-2}^{0, \beta}\left(\bar{B}_{r_{\varepsilon, \beta}}\right)} \\
& \leq c_{\kappa} r_{\varepsilon, \beta}^{2} .
\end{aligned}
$$

Then the first estimate of part $i$ ) follows. On the other hand, we have for $\left\|v_{i}\right\|_{\mathcal{C}_{\mu}^{2, \beta}\left(B_{r_{\varepsilon, \beta}}\right)} \leq 2 c_{\kappa} r_{\varepsilon, \beta}$

$$
\begin{aligned}
& \left\|\mathcal{N}\left(v_{2}\right)-\mathcal{N}\left(v_{1}\right)\right\|_{\mathcal{C}_{\mu}^{2, \alpha}\left(\mathbb{R}^{2}\right)} \\
& \leq c_{\kappa}\left(\varepsilon^{1 /(\beta+1)}+\varepsilon^{\mu /(\beta+1)} r_{\varepsilon, \beta}+\varepsilon^{4(1-\gamma)}+\varepsilon^{4(1-\mu)}\left(1+\varepsilon^{1 /(\beta+1)}\right)\right)\left\|v_{2}-v_{1}\right\|_{\mathcal{E}_{\mu}^{2, \alpha}\left(\mathbb{R}^{2}\right)} \\
& \leq c_{\kappa} r_{\varepsilon, \beta}\left\|v_{2}-v_{1}\right\|_{\mathcal{C}_{\mu}^{2, \alpha}\left(\mathbb{R}^{2}\right)} .
\end{aligned}
$$

This yields the second estimate of part $i$ ).

Observe that these estimates are uniform in $\tau$ provided $\tau$ remains in a fixed compact subset of $(0, \infty)$. Applying a contraction mapping argument, we obtain the following proposition.

Proposition 4. Given $\beta \notin \mathbb{N}, \mu \in(0,1-\gamma]$ and $\kappa>0$, there exists $\varepsilon_{\kappa}>0$, $\lambda_{\kappa}>0$, (depending on $\kappa$ ) such that for all $\varepsilon \in\left(0, \varepsilon_{\kappa}\right), \lambda \in\left(0, \lambda_{\kappa}\right)$ satisfying $\left(H_{\lambda}\right)$, for all $\tau$ in some fixed compact subset of $\left[\tau_{-}, \tau^{+}\right] \subset(0, \infty)$ and for a given $\varphi$ satisfying (20) and (27), then there exists a unique $v_{\beta}\left(=\bar{v}_{\varepsilon, \tau, \beta, \varphi}\right)$ solution of (26) satisfying

$$
\left\|v_{\beta}\right\|_{\mathcal{C}_{\mu}^{2, \alpha}\left(B_{r_{\varepsilon, \beta}}\right)} \leq 2 c_{\kappa} r_{\varepsilon, \beta} .
$$

Notice that it follows from this proposition that the function

$$
\mathbf{v}+\bar{v}_{\varepsilon, \tau, \beta, \varphi}=u_{\varepsilon, \tau, \beta}+h-\log K(0)+H^{i}\left(\cdot / r_{\varepsilon, \beta}\right)+\bar{v}_{\varepsilon, \tau, \beta, \varphi}
$$


solves (25) in $B_{r_{\varepsilon, \beta}}$. Observe that the function $\bar{v}_{\varepsilon, \tau, \beta, \varphi}$ obtained as a fixed point for contraction mapping, it depends smoothly on the parameter $\tau$ and the boundary data $\varphi$.

We turn now to the case when 0 is not a zero of $V$, which corresponds to the case $\beta=0$.

Proposition 5. Given $\kappa>0, \mu \in(1,2)$ and $\beta=0$, then there exists $\varepsilon_{\kappa}>0$, $\lambda_{\kappa}>0$ (depending on $\kappa$ ) such that for all $\varepsilon \in\left(0, \varepsilon_{\kappa}\right), \lambda \in\left(0, \lambda_{\kappa}\right)$ satisfying $\left(C_{\lambda}\right)$, for all $\tau$ in some fixed compact subset of $\left[\tau_{-}, \tau^{+}\right] \subset(0, \infty)$ and for a given $\varphi$ satisfying (21) and (28), then there exists a unique $v\left(=\bar{v}_{\varepsilon, \tau, \varphi}\right)$ solution of $(26)_{\mid \beta=0}$ such that

$$
\|v\|_{\mathcal{C}_{\mu}^{2, \alpha}\left(\mathbb{R}^{2}\right)} \leq 2 c_{\kappa} r_{\varepsilon}^{2}
$$

\section{The nonlinear exterior problem}

Denote by

$$
r_{\varepsilon, \beta_{i}}=\max \left(\varepsilon^{2(1-\gamma)-\delta / 2\left(\beta_{i}+1\right)}, \lambda^{1 / 2} \varepsilon^{-\delta / 2\left(\beta_{i}+1\right)}, \lambda^{1 / 2}, \varepsilon^{2 /\left(2 \beta_{i}+3\right)}\right) .
$$

and $r_{\varepsilon}=\max \left(\varepsilon^{2(1-\gamma)-\delta / 2}, \lambda^{1 / 2} \varepsilon^{-\delta / 2}, \lambda^{1 / 2}, \varepsilon^{1 / 2}\right)$ and recall that $\bar{\beta}=\min _{1 \leq i \leq N} \beta_{i}$. Let $G(\cdot, \tilde{x})$ be the unique solution of

$$
-\Delta G(\cdot, \tilde{x})=8 \pi \delta_{\tilde{x}}
$$

in $\Omega$, with $G(\cdot, \tilde{x})=0$ on $\partial \Omega$. Recall that the following decomposition holds

$$
G(x, \tilde{x})=-4 \log |x-\tilde{x}|+H(x, \tilde{x})
$$

where $x \longmapsto H(x, \tilde{x})$ is a smooth function. Here we give an estimate of the gradient of $H(x, \tilde{x})$ without proof (see [27] and more details in [22], Lemma 2.1 ), there exists a constant $c>0$, so that

$$
\left|\nabla_{x} H(x, \tilde{x})\right| \leq c \log |x-\tilde{x}|
$$

Let $\tilde{\eta}:=\left(\tilde{\eta}^{1}, \ldots, \tilde{\eta}^{K}\right) \in \mathbb{R}^{K}$ close to $0, \tilde{\varphi}:=\left(\tilde{\varphi}^{1}, \ldots, \tilde{\varphi}^{K}\right) \in\left(\mathcal{C}^{2, \alpha}\left(S^{1}\right)\right)^{K}$ satisfying $(23)$ and $\tilde{\mathbf{q}}:=\left(p_{j_{1}}, \ldots, p_{j_{s}}, \tilde{q}_{1}, \ldots, \tilde{q}_{m}\right)$ close to $\mathbf{q}:=\left(p_{j_{1}}, \ldots, p_{j_{s}}, q_{1}, \ldots, q_{m}\right)$. Note that the set $\left\{q_{1}, \ldots, q_{m}\right\}$ can be empty . 
We define

$$
\tilde{\mathbf{v}}:=\left\{\begin{aligned}
\bullet & \sum_{i=1}^{s}\left(1+\beta_{j_{i}}+\tilde{\eta}^{j_{i}}\right) G\left(\cdot, p_{j_{i}}\right)+\sum_{i=1}^{s} \chi_{r_{0}}\left(\cdot-p_{j_{i}}\right) H^{e}\left(\tilde{\varphi}^{j_{i}} ;\left(\cdot-p_{j_{i}}\right) / r_{\varepsilon, \beta_{j_{i}}}\right) \\
& \text { if } S \cap \Lambda=S=\left\{p_{j_{1}}, \cdots, p_{j_{s}}\right\}, \\
\bullet & \sum_{i=1}^{s}\left(1+\beta_{j_{i}}+\tilde{\eta}^{j_{i}}\right) G\left(\cdot, p_{j_{i}}\right)+\sum_{i=1}^{s} \chi_{r_{0}}\left(\cdot-p_{j_{i}}\right) H^{e}\left(\tilde{\varphi}^{j_{i}} ;\left(\cdot-p_{j_{i}}\right) / r_{\varepsilon, \beta_{j_{i}}}\right) \\
& +\sum_{i=1}^{m}\left(1+\tilde{\eta}^{i}\right) G\left(\cdot, \tilde{q}_{i}\right)+\sum_{i=1}^{m} \chi_{r_{0}}\left(\cdot-\tilde{q}_{i}\right) H^{e}\left(\tilde{\varphi}^{i} ;\left(\cdot-\tilde{q}_{i}\right) / r_{\varepsilon}\right) \\
& \text { if } S \cap \Lambda=\left\{p_{j_{1}}, \cdots, p_{j_{s}}\right\} \text { and } S \backslash \Lambda=\left\{q_{1}, \cdots, q_{m}\right\}, \text { with } s+m=K,
\end{aligned}\right.
$$

where $\chi_{r_{0}}$ is a cutoff function identically equal to 1 in $B_{r_{0} / 2}$ and identically equal to 0 outside $B_{r_{0}}$. We suppose that

$$
\left\{\begin{array}{lll}
\tilde{q}_{i}=q_{i}=p_{j_{i}} & \text { if } & q_{i} \in S \cap \Lambda \\
\left|\tilde{q}_{i}-q_{i}\right|<\kappa r_{\varepsilon} & \text { if } \quad & q_{i} \in S \backslash \Lambda
\end{array}\right.
$$

and we let

$$
\begin{cases}\beta_{j_{i}}=\beta_{i} & \text { if } q_{i} \in S \cap \Lambda \\ \beta_{i}=0 & \text { if } q_{i} \in S \backslash \Lambda .\end{cases}
$$

We would like to find a solution of

$$
\Delta v+\lambda|\nabla v|^{2}+\varepsilon^{2} \prod_{i=1}^{N}\left|x-p_{i}\right|^{2 \beta_{i}} f(x)\left(e^{v}+e^{\gamma v}\right)=0
$$

in $\bar{\Omega}_{r_{\varepsilon, \bar{\beta}}}(\tilde{\mathbf{q}}):=\bar{\Omega}-\cup_{i=1}^{K} B_{r_{\varepsilon, \bar{\beta}}}\left(\tilde{q}_{i}\right)$ which is a perturbation of $\tilde{\mathbf{v}}$. Writing $v=$ $\tilde{\mathbf{v}}+\tilde{v}$, this amounts to solve

$$
-\Delta \tilde{v}=\varepsilon^{2} \prod_{i=1}^{N}\left|x-p_{i}\right|^{2 \beta_{i}} f(x)\left(e^{\tilde{\mathbf{v}}+\tilde{v}}+e^{\gamma \tilde{\mathbf{v}}+\gamma \tilde{v}}\right)+\Delta \tilde{\mathbf{v}}+\lambda|\nabla(\tilde{\mathbf{v}}+\tilde{v})|^{2} .
$$

We need to define some auxiliary weighted spaces :

Definition 4. Let $\bar{r} \in\left(0, r_{0} / 2\right), k \in \mathbb{R}, \alpha \in(0,1)$ and $\nu \in \mathbb{R}$, we define the Hölder weighted space $\mathcal{C}_{\nu}^{k, \alpha}\left(\bar{\Omega}_{\bar{r}}(\mathbf{q})\right)$ as the set of functions $w \in \mathcal{C}^{k, \alpha}\left(\bar{\Omega}_{\bar{r}}(\mathbf{q})\right)$ for which the following norm

$\|w\|_{\mathcal{C}_{\nu}^{k, \alpha}\left(\bar{\Omega}_{\bar{r}}(\mathbf{q})\right)}:=\|w\|_{\mathcal{C}^{k, \alpha}\left(\bar{\Omega}_{r_{0} / 2}(\mathbf{q})\right)}+\sum_{i=1}^{K} \sup _{r \in\left[\bar{r}, r_{0} / 2\right)}\left(r^{-\nu}\left\|w\left(q_{i}+r \cdot\right)\right\|_{\mathcal{C}^{k, \alpha}\left(\bar{B}_{2}-B_{1}\right)}\right)$.

is finite. 
For all $\sigma \in\left(0, r_{0} / 2\right)$ and all $Y=\left(y_{1}, \cdots, y_{m}\right) \in \Omega^{m}$ such that $\|X-Y\| \leq$ $r_{0} / 2$, where $X=\left(x_{1}, \cdots, x_{m}\right)$, we denote by

$$
\tilde{\varepsilon}_{\sigma, Y}: \mathcal{C}_{\nu}^{0, \alpha}\left(\bar{\Omega}_{\sigma}(Y)\right) \longrightarrow \mathcal{C}_{\nu}^{0, \alpha}\left(\bar{\Omega}^{*}(Y)\right),
$$

the extension operator defined by $\tilde{\mathcal{E}}_{\sigma, Y}(f)=f$ in $\bar{\Omega}_{\sigma}(Y)$

$$
\tilde{\mathcal{E}}_{\sigma, Y}(f)\left(y_{i}+x\right)=\tilde{\chi}\left(\frac{|x|}{\sigma}\right) f\left(y_{i}+\sigma \frac{x}{|x|}\right)
$$

in $B_{\sigma}\left(y_{i}\right)-B_{\sigma / 2}\left(y_{i}\right)$, for each $i=1, \ldots, m$ and $\tilde{\mathcal{E}}_{\sigma, Y}(f)=0$ in each $B_{\sigma / 2}\left(y_{i}\right)$, where $t \longmapsto \tilde{\chi}(t)$ is a cutoff function identically equal to 1 for $t \geq 1$ and identically equal to 0 for $t \leq 1 / 2$. It is easy to check that there exists a constant $c=c(\nu)>0$ only depending on $\nu$ such that

$$
\left\|\tilde{\mathcal{E}}_{\sigma, Y}(w)\right\|_{\mathcal{C}_{\nu}^{0, \alpha}\left(\bar{\Omega}^{*}(Y)\right)} \leq c\|w\|_{\mathcal{C}_{\nu}^{0, \alpha}\left(\bar{\Omega}_{\sigma}(Y)\right)} .
$$

Fix $\nu \in(-1,0)$ and denote by $\tilde{\mathfrak{G}}_{\nu}: \mathcal{C}_{\nu-2}^{0, \alpha}\left(\bar{\Omega}^{*}(\tilde{\mathbf{q}})\right) \longrightarrow \mathcal{C}_{\nu}^{2, \alpha}\left(\bar{\Omega}^{*}(\tilde{\mathbf{q}})\right)$ the right inverse of $\Delta$ provided by Proposition 3 with $\bar{\Omega}^{*}(\tilde{\mathbf{q}})=\bar{\Omega}-\left\{\tilde{q}_{1}, \ldots, \tilde{q}_{K}\right\}$. Clearly, it is enough to find $\tilde{v} \in \mathcal{C}_{\nu}^{2, \alpha}\left(\bar{\Omega}^{*}(\tilde{\mathbf{q}})\right)$ solution of

$$
\tilde{v}=\tilde{\mathcal{G}}_{\nu} \circ \tilde{\mathcal{E}}_{r_{\varepsilon, \beta}, \tilde{\mathbf{q}}} \circ \tilde{\mathfrak{R}}(\tilde{v}),
$$

where

$$
\tilde{\mathfrak{R}}(\tilde{v})=\varepsilon^{2} \prod_{i=1}^{N}\left|x-p_{i}\right|^{2 \beta_{i}} f(x)\left(e^{\tilde{\mathbf{v}}+\tilde{v}}+e^{\gamma \tilde{\mathbf{v}}+\gamma \tilde{v}}\right)+\lambda|\nabla(\tilde{\mathbf{v}}+\tilde{v})|^{2}+\Delta \tilde{\mathbf{v}} .
$$

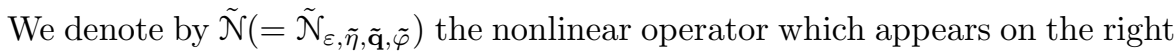
hand side of equation (34).

Given $\kappa>0$ (whose value will be fixed later on), we further assume that when $S \cap \Lambda=S$, for $i=1, \ldots, s$ the functions $\tilde{\varphi}^{j_{i}}$ and the parameters $\tilde{\eta}^{j_{i}}$ satisfy

$$
\left\|\tilde{\varphi}^{j_{i}}\right\|_{\mathrm{C}^{2, \alpha}} \leq \kappa r_{\varepsilon, \beta_{j_{i}}}
$$

and

$$
\left|\tilde{\eta}^{j_{i}}\right| \leq \kappa r_{\varepsilon, \beta_{j_{i}}}
$$

and when $S \cap \Lambda=\left\{p_{j_{1}}, \cdots, p_{j_{s}}\right\}$ and $S \backslash \Lambda=\left\{q_{1}, \cdots, q_{m}\right\}$, we assume that for $i=1, \ldots, s$ the functions $\tilde{\varphi}^{j_{i}}$ and the parameters $\tilde{\eta}^{j_{i}}$ satisfy

$$
\left\|\tilde{\varphi}^{j_{i}}\right\|_{\mathcal{C}^{2, \alpha}} \leq \kappa r_{\varepsilon, \beta_{j_{i}}}
$$


and

$$
\left|\tilde{\eta}^{j_{i}}\right| \leq \kappa r_{\varepsilon, \beta_{j_{i}}}
$$

also for $i=1, \ldots, m$ the functions $\tilde{\varphi}^{i}$ and the parameters $\tilde{\eta}^{i}$ satisfy

$$
\left\|\tilde{\varphi}^{i}\right\|_{\mathcal{C}^{2, \alpha}} \leq \kappa r_{\varepsilon}^{2}
$$

and

$$
\left|\tilde{\eta}^{i}\right| \leq \kappa r_{\varepsilon}^{2}
$$

Then, the following result holds.

Lemma 5. Under the above assumptions, given $\kappa>1$, there exists a constant $c_{\kappa}>1$ such that

$$
\|\tilde{\mathcal{N}}(0)\|_{\mathcal{C}_{\nu}^{2, \alpha}\left(\bar{\Omega}^{*}(\tilde{\mathbf{q}})\right)} \leq c_{\kappa} r_{\varepsilon, \bar{\beta}}
$$

and

$$
\left\|\tilde{\mathcal{N}}\left(\tilde{v}_{2}\right)-\tilde{\mathcal{N}}\left(\tilde{v}_{1}\right)\right\|_{\mathcal{C}_{\nu}^{2, \alpha}\left(\bar{\Omega}^{*}(\tilde{\mathbf{q}})\right)} \leq c_{\kappa} r_{\varepsilon, \bar{\beta}}\left\|\tilde{v}_{2}-\tilde{v}_{1}\right\|_{\mathcal{C}_{\nu}^{2, \alpha}\left(\bar{\Omega}^{*}(\tilde{\mathbf{q}})\right)}
$$

provided $\tilde{v}_{1}, \tilde{v}_{2} \in \mathcal{C}_{\nu}^{2, \alpha}\left(\bar{\Omega}^{*}(\tilde{\mathbf{q}})\right)$ and satisfy $\left\|\tilde{v}_{i}\right\|_{\mathcal{C}_{\nu}^{2, \alpha}\left(\bar{\Omega}^{*}(\tilde{\mathbf{q}})\right)} \leq 2 c_{\kappa} r_{\varepsilon, \bar{\beta}}$.

Proof . Recall that $\tilde{\mathcal{N}}(\tilde{v})=\tilde{\mathcal{G}}_{\nu, \beta} \circ \tilde{\mathfrak{R}}(\tilde{v})$, we will estimate $\tilde{\mathcal{N}}(0)$ in different subregions of $\bar{\Omega}^{*}(\tilde{\mathbf{q}})$.

- If $S \cap \Lambda=S$ we have

* In $B_{r_{0}}\left(p_{j_{i}}\right)$, we have $\chi_{r_{0}}\left(x-p_{j_{i}}\right)=1$ and $\Delta \tilde{\mathbf{v}}=0$ then

$$
\left|H^{e}\left(\tilde{\varphi}^{j_{i}} ;\left(x-\tilde{p}_{j_{i}}\right) / r_{\varepsilon, \beta_{j_{i}}}\right)\right| \leq \kappa r_{\varepsilon, \beta_{j_{i}}}^{2} r^{-1}
$$

so there exist a constant $c_{\kappa}^{(i)}>0, i=1,2$ and $c_{\kappa}>0$ such that

$$
\begin{aligned}
& |\tilde{\mathfrak{R}}(0)| \leq c_{\kappa}^{(1)} \varepsilon^{2}|f(x)|\left|x-p_{j_{i}}\right|^{2 \beta_{j_{i}}}\left|x-p_{j_{i}}\right|^{-4\left(1+\beta_{j_{i}}+\tilde{\eta}^{j_{i}}\right)} \prod_{\ell=1, \ell \neq i}^{N}\left|x-p_{j_{\ell}}\right|^{2 \beta_{j_{\ell}} \times} \\
& \quad \prod_{\ell=1, \ell \neq i}^{m}\left|x-p_{j_{\ell}}\right|^{-4\left(1+\beta_{j_{\ell}}+\tilde{\eta}^{j_{\ell}}\right)}+c_{\kappa}^{(2)} \lambda\left(\left(1+\beta_{j_{i}}+\tilde{\eta}^{j_{i}}\right)\left(r^{-1}+\log r\right)+r_{\varepsilon, \beta_{j_{i}}}^{2} r^{-2}\right)^{2} \\
& \leq c_{\kappa}\left(\varepsilon^{2} r^{-2 \beta_{j_{i}}-4\left(1+\tilde{\eta}^{j_{i}}\right)}+\lambda\left(\left(1+\beta_{j_{i}}+\tilde{\eta}^{j_{i}}\right)\left(r^{-1}+\log r\right)+r_{\varepsilon, \beta_{j_{i}}}^{2} r^{-2}\right)^{2}\right) .
\end{aligned}
$$

Hence, for $\nu \in(-1,0), \tilde{\eta}^{j_{i}}$ small enough, there exist a constant $\bar{c}_{\kappa}>0$ such that

$$
\|\tilde{\mathfrak{R}}(0)\|_{\mathcal{C}_{\nu-2}^{0, \alpha}\left(\bigcup_{i=1}^{s} B_{r_{0}}\left(p_{j_{i}}\right)\right)} \leq \sup _{r_{\varepsilon, \bar{\beta}} \leq r \leq r_{0} / 2} r^{2-\nu}|\tilde{\mathfrak{R}}(0)| \leq \bar{c}_{\kappa} r_{\varepsilon, \bar{\beta}}
$$


$*$ In $\Omega-B_{r_{0}}\left(p_{j_{i}}\right)$, we have $\chi_{r_{0}}\left(x-p_{j_{i}}\right)=0$ and $\Delta \tilde{\mathbf{v}}=0$. Thus

$$
\begin{aligned}
|\tilde{\mathfrak{R}}(0)| & \leq c_{\kappa} \varepsilon^{2}|f(x)| \prod_{\ell=1}^{N}\left|x-p_{j_{\ell}}\right|^{2 \beta_{j_{\ell}}} \prod_{\ell=1}^{m} e^{\left(1+\beta_{j_{\ell}}+\tilde{\eta}^{j_{\ell}}\right) G\left(x, p_{j_{\ell}}\right)} \\
& +c_{\kappa} \lambda\left(\left(1+\beta_{j_{i}}+\tilde{\eta}^{j_{i}}\right) r^{-1}+\left(1+\beta_{j_{i}}+\tilde{\eta}^{j_{i}}\right) \log r+r_{\varepsilon, \beta_{j_{i}}}^{2} r^{-2}\right)^{2} .
\end{aligned}
$$

So,

$$
\|\tilde{\Re}(0)\|_{\mathcal{C}_{\nu-2}^{0, \alpha}\left(\Omega-\bigcup_{i=1}^{s} B_{r_{0}}\left(p_{j_{i}}\right)\right)} \leq \sup _{r_{0} \leq r} r^{2-\nu}|\tilde{\mathfrak{R}}(0)| \leq c_{\kappa} \varepsilon^{2}+c_{\kappa} \lambda
$$

* In $B_{r_{0}}\left(p_{j_{i}}\right)-B_{r_{0} / 2}\left(p_{j_{i}}\right)$, we have $\left|H^{e}\left(\tilde{\varphi}_{j_{i}} ;\left(x-p_{j_{i}}\right) / r_{\varepsilon, \beta_{j_{i}}}\right)\right| \leq \kappa r_{\varepsilon, \beta_{j_{i}}}^{2} r^{-1}$. Consequently,

$$
\begin{aligned}
|\tilde{\mathfrak{R}}(0)| & \leq c_{\kappa} \varepsilon^{2} r^{-2 \beta_{j_{i}}-4\left(1+\beta_{j_{i}}+\tilde{\eta}^{j_{i}}\right)}+c_{\kappa} \lambda\left(\left(1+\beta_{j_{i}}+\tilde{\eta}^{j_{i}}\right)\left(r^{-1}+\log r\right)+r_{\varepsilon, \beta_{j_{i}}}^{2} r^{-2}\right)^{2} \\
& +\sum_{i=1}^{s}\left|\left[\Delta, \chi_{r_{0}}\left(x-p_{j_{i}}\right)\right]\right|\left|H^{e}\left(\tilde{\varphi}_{j_{i}} ;\left(x-p_{j_{i}}\right) / r_{\varepsilon, \beta_{j_{i}}}\right)\right| \\
& \leq c_{\kappa}\left[\varepsilon^{2}+\lambda\left(\left(1+\beta_{j_{i}}+\tilde{\eta}^{j_{i}}\right)\left(r^{-1}+\log r\right)+r_{\varepsilon, \beta_{j_{i}}}^{2} r^{-2}\right)^{2}+r_{\varepsilon, \beta_{j_{i}}}^{2} r^{-1}\right]
\end{aligned}
$$

where

$$
\left[\Delta, \chi_{r_{0}}\right] w=\Delta w \chi_{r_{0}}+w \Delta \chi_{r_{0}}+2 \nabla w \cdot \nabla \chi_{r_{0}}
$$

Then, we get

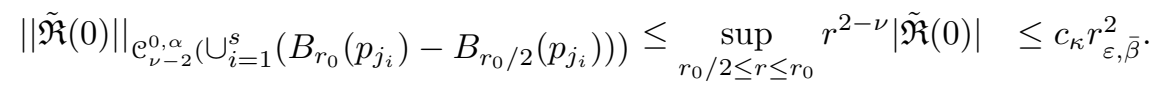

Finally,

$$
\|\tilde{\Re}(0)\|_{\mathcal{C}_{\nu-2}^{0, \alpha}}\left(\Omega-\cup_{i=1}^{s} B_{r_{0}}\left(p_{j_{i}}\right)\right) \leq c_{\kappa} r_{\varepsilon, \bar{\beta}}
$$

- If $S \cap \Lambda=\left\{p_{j_{1}}, \cdots, p_{j_{s}}\right\}$ and $S \backslash \Lambda=\left\{q_{1}, \cdots, q_{m}\right\}$, then

* If $x \in B_{r_{\varepsilon, \beta_{j_{i}}}}\left(p_{j_{i}}\right)$, then we get the same estimates as above taking in to account that $\tilde{\mathfrak{R}}(0)$ is uniformly bounded in neighborhood of $S \backslash \Gamma$ and using the expansion of $\tilde{\mathbf{v}}$ given by (29), then we get the estimate (42).

$\star$ If $x \in B_{r_{\varepsilon}}\left(\tilde{q}_{i}\right)$ then

* In $B_{r_{0}}\left(\tilde{q}_{i}\right)$, we have $\chi_{r_{0}}\left(x-\tilde{q}_{i}\right)=1$ and $\Delta \tilde{\mathbf{v}}=0$ and

$$
\left|H^{e}\left(\tilde{\varphi}_{i} ;\left(x-\tilde{x}_{i}\right) / r_{\varepsilon}\right)\right| \leq \kappa r_{\varepsilon}^{3} r^{-1},
$$


so that

$$
\begin{aligned}
|\tilde{\mathfrak{R}}(0)| & \leq c_{\kappa} \varepsilon^{2}|f(x)|\left|x-\tilde{q}_{i}\right|^{-4\left(1+\tilde{\eta}^{i}\right)} \prod_{\ell=1, \ell \neq i}^{m}\left|x-\tilde{q}_{\ell}\right|^{-4\left(1+\tilde{\eta}^{\ell}\right)} \\
& +c_{\kappa} \lambda\left(\left(1+\tilde{\eta}^{i}\right)\left(r^{-1}+\log r\right)+r_{\varepsilon}^{3} r^{-2}\right)^{2} \\
& \leq c_{\kappa} \varepsilon^{2} r^{-4\left(1+\tilde{\eta}^{i}\right)}+c_{\kappa} \lambda\left(\left(1+\tilde{\eta}^{i}\right)\left(r^{-1}+\log r\right)+r_{\varepsilon}^{3} r^{-2}\right)^{2} .
\end{aligned}
$$

Hence, for $\nu \in(-1,0)$ and for $\tilde{\eta}^{i}$ small enough, we get

$$
\|\tilde{\mathfrak{R}}(0)\|_{\mathcal{C}_{\nu-2}^{0, \alpha}\left(\bigcup_{i=1}^{m} B_{r_{0}}\left(\tilde{q}_{i}\right)\right)} \leq \sup _{r_{\varepsilon} \leq r \leq r_{0} / 2} r^{2-\nu}|\tilde{\mathfrak{R}}(0)| \leq c_{\kappa} r_{\varepsilon}^{2}+c_{\kappa} \lambda .
$$

$*$ In $\Omega-B_{r_{0}}\left(\tilde{q}_{i}\right)$, we have $\chi_{r_{0}}\left(x-\tilde{q}_{i}\right)=0$ and $\Delta \tilde{\mathbf{v}}=0$. Thus

$$
|\tilde{\mathfrak{R}}(0)| \leq c_{\kappa} \varepsilon^{2}|f(x)| \prod_{\ell=1}^{m} e^{\left(1+\tilde{\eta}^{\ell}\right) G\left(x, q_{\ell}\right)}+c_{\kappa} \lambda\left(\left(1+\tilde{\eta}^{i}\right)\left(r^{-1}+\log r\right)+r_{\varepsilon}^{3} r^{-2}\right)^{2} .
$$

So

$$
\|\tilde{\mathfrak{R}}(0)\|_{\mathcal{C}_{\nu-2}^{0, \alpha}\left(\Omega-\bigcup_{i=1}^{m} B_{r_{0}}\left(\tilde{q}_{i}\right)\right)} \leq \sup _{r_{0} \leq r} r^{2-\nu}|\tilde{\mathfrak{R}}(0)| \leq c_{\kappa} \varepsilon^{2}+c_{\kappa} \lambda .
$$

* In $B_{r_{0}}\left(\tilde{q}_{i}\right)-B_{r_{0} / 2}\left(\tilde{q}_{i}\right)$, we have $\left|H^{e}\left(\tilde{\varphi}_{i} ;\left(x-\tilde{q}_{i}\right) / r_{\varepsilon}\right)\right| \leq \kappa r_{\varepsilon}^{3} r^{-1}$. Consequently,

$$
\begin{aligned}
|\tilde{\mathfrak{R}}(0)| & \leq c_{\kappa} \varepsilon^{2} r^{-4\left(1+\tilde{\eta}^{j_{i}}\right)}+c_{\kappa} \lambda\left(\left(1+\tilde{\eta}^{i}\right)\left(r^{-1}+\log r\right)+r_{\varepsilon}^{3} r^{-2}\right)^{2} \\
& +\sum_{i=1}^{m}\left|\left[\Delta, \chi_{r_{0}}\left(x-\tilde{q}_{i}\right)\right]\right|\left|H^{e}\left(\tilde{\varphi}_{i} ;\left(x-\tilde{q}_{i}\right) / r_{\varepsilon, \beta_{i}}\right)\right| \\
& \leq c_{\kappa}\left(\varepsilon^{2}+\lambda\left(\left(1+\tilde{\eta}^{i}\right)\left(r^{-1}+\log r\right)+r_{\varepsilon}^{3} r^{-2}\right)^{2}+r^{-1} r_{\varepsilon}^{2}\right) .
\end{aligned}
$$

Then

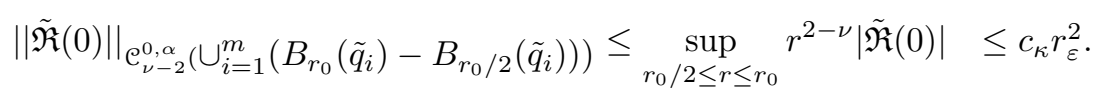

So,

$$
\|\tilde{\mathfrak{R}}(0)\|_{\mathcal{C}_{\nu-2}^{0, \alpha}\left(\cup_{i=1}^{m}\left(\Omega-B_{r_{0}}\left(\tilde{q}_{i}\right)\right)\right)} \leq c_{\kappa} r_{\varepsilon}^{2} .
$$

Then by (42) and (45), we get

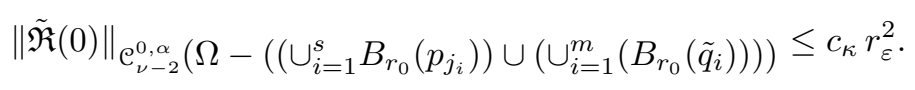

Finally by (33), we get

$$
\|\tilde{\mathcal{N}}(0)\|_{\mathcal{C}_{\nu}^{2, \alpha}\left(\bar{\Omega}^{*}(\mathbf{p} \cup \mathbf{q})\right)} \leq c_{\kappa} r_{\varepsilon, \bar{\beta}}
$$


For the proof of the second estimate let $\tilde{v}_{1}$ and $\tilde{v}_{2} \in C_{\nu}^{2, \alpha}\left(\bar{\Omega}^{*}(\tilde{\mathbf{q}})\right)$, then we have

$$
\begin{aligned}
\left|\tilde{\mathfrak{R}}\left(\tilde{v}_{2}\right)-\tilde{\mathfrak{R}}\left(\tilde{v}_{1}\right)\right| \leq & c_{\kappa} \varepsilon^{2}\left|x-p_{j_{i}}\right|^{2 \beta_{j_{i}}} \prod_{\substack{\ell=1, \ell \neq i\\
}}^{N}\left|x-p_{j_{\ell}}\right|^{2 \beta_{j_{\ell}}}|f(x)| e^{\tilde{\mathbf{v}}}\left(e^{\tilde{v}_{2}}-e^{\tilde{v}_{1}}\right) \mid \\
& +\left.c_{\kappa} \lambda|| \nabla\left(\tilde{\mathbf{v}}+\tilde{v}_{2}\right)\right|^{2}-\left|\nabla\left(\tilde{\mathbf{v}}+\tilde{v}_{1}\right)\right|^{2} \mid .
\end{aligned}
$$

Then

- If $S \cap \Lambda=S$ we have that

$$
\begin{aligned}
& \left|\tilde{\mathfrak{R}}\left(\tilde{v}_{2}\right)-\tilde{\mathfrak{R}}\left(\tilde{v}_{1}\right)\right| \leq c_{\kappa} \varepsilon^{2}\left|x-p_{j_{i}}\right|^{2 \beta_{j_{i}}}\left|x-p_{j_{i}}\right|^{-4\left(1+\beta_{j_{i}}+\tilde{\eta}^{j_{i}}\right)}\left|\tilde{v}_{2}-\tilde{v}_{1}\right| \\
& +c_{\kappa} \lambda\left|\nabla\left(\tilde{v}_{2}-\tilde{v}_{1}\right)\right|\left(\left|\nabla\left(\tilde{v}_{2}+\tilde{v}_{1}\right)\right|+2|\nabla \tilde{\mathbf{v}}|\right) \\
& \leq c_{\kappa} \varepsilon^{2} r^{-2 \beta_{j_{i}}-4\left(1+\tilde{\eta}^{j_{i}}\right)}\left|\tilde{v}_{2}-\tilde{v}_{1}\right|+c_{\kappa} \lambda\left|\nabla\left(\tilde{v}_{2}-\tilde{v}_{1}\right)\right|\left(\left|\nabla\left(\tilde{v}_{2}+\tilde{v}_{1}\right)\right|+2|\nabla \tilde{\mathbf{v}}|\right) .
\end{aligned}
$$

Hence, for $\tilde{\eta}^{j_{i}}$ small enough, we get

$$
\left\|\tilde{\mathcal{N}}\left(\tilde{v}_{2}\right)-\tilde{\mathcal{N}}\left(\tilde{v}_{1}\right)\right\|_{\mathcal{C}_{\nu}^{2, \alpha}\left(\bar{\Omega}^{*}(\mathbf{p})\right)} \leq c_{\kappa} r_{\varepsilon, \bar{\beta}}\left\|\tilde{v}_{2}-\tilde{v}_{1}\right\|_{\mathcal{C}_{\nu}^{2, \alpha}\left(\bar{\Omega}^{*}(\mathbf{p})\right)} .
$$

- If $S \cap \Lambda=\left\{p_{j_{1}}, \cdots, p_{j_{s}}\right\}$ and $S \backslash \Lambda=\left\{q_{1}, \cdots, q_{m}\right\}$ then

$\star$ If $x \in B_{r_{\varepsilon, \beta_{j_{i}}}}\left(p_{j_{i}}\right)$, then we get the estimate (46).

$\star$ If $x \in B_{r_{\varepsilon}}\left(\tilde{q}_{i}\right)$ then

$$
\begin{aligned}
& \left|\tilde{\mathfrak{R}}\left(\tilde{v}_{2}\right)-\tilde{\mathfrak{R}}\left(\tilde{v}_{1}\right)\right| \leq c_{\kappa} \varepsilon^{2}\left|x-q_{i}\right|^{-4\left(1+\tilde{\eta}^{i}\right)}\left|\tilde{v}_{2}-\tilde{v}_{1}\right|+ \\
& c_{\kappa} \lambda\left|\nabla\left(\tilde{v}_{2}-\tilde{v}_{1}\right)\right|\left(\left|\nabla\left(\tilde{v}_{2}+\tilde{v}_{1}\right)\right|+2|\nabla \tilde{\mathbf{v}}|\right) \\
& \leq c_{\kappa} \varepsilon^{2} r^{-4\left(1+\tilde{\eta}^{j_{i}}\right)}\left|\tilde{v}_{2}-\tilde{v}_{1}\right|+c_{\kappa} \lambda\left|\nabla\left(\tilde{v}_{2}-\tilde{v}_{1}\right)\right|\left(\left|\nabla\left(\tilde{v}_{2}+\tilde{v}_{1}\right)\right|+2|\nabla \tilde{\mathbf{v}}|\right) .
\end{aligned}
$$

So, for $\tilde{\eta}^{j_{i}}$ small enough, we get

$$
\left\|\tilde{\mathcal{N}}\left(\tilde{v}_{2}\right)-\tilde{\mathcal{N}}\left(\tilde{v}_{1}\right)\right\|_{\mathcal{C}_{\nu}^{2, \alpha}\left(\bar{\Omega}^{*}(\tilde{\mathbf{q}})\right)} \leq c_{\kappa} r_{\varepsilon}^{2}\left\|\tilde{v}_{2}-\tilde{v}_{1}\right\|_{\mathcal{C}_{\nu}^{2, \beta}\left(\bar{\Omega}^{*}(\tilde{\mathbf{q}})\right)} .
$$

Then by (46) and (47), we get the desired result. that

Applying a fixed point Theorem for contraction mappings, we conclude

Proposition 6. Given $\kappa>1$, there exists $\varepsilon_{\kappa}>0$ and $\lambda_{\kappa}>0$, (depending on $\kappa)$ such that for all $\varepsilon \in\left(0, \varepsilon_{\kappa}\right), \lambda \in\left(0, \lambda_{\kappa}\right)$, for all set of parameters $\tilde{\eta}^{j_{i}}$ satisfying (36) and functions $\tilde{\varphi}$ satisfying (23), there exists a unique $\tilde{v}(=$

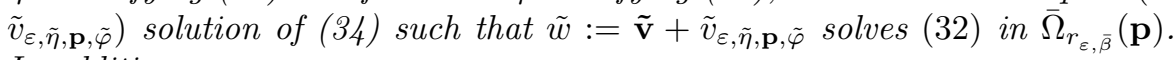
In addition

$$
\|\tilde{v}\|_{\mathcal{C}_{\nu}^{2, \alpha}\left(\bar{\Omega}^{*}(\mathbf{p})\right)} \leq 2 c_{\kappa} r_{\varepsilon, \bar{\beta}} .
$$


As in the previous section, observe that the function $\tilde{v}_{\varepsilon, \tilde{\eta}, \mathbf{p}, \tilde{\varphi}}$ being obtained as a fixed point for contraction mapping, depends smoothly on the parameters $\tilde{\eta}^{j_{i}}$ for $i=1, \ldots, s$ and the boundary data $\tilde{\varphi}$.

Proposition 7. Given $\kappa>1$, there exists $\varepsilon_{\kappa}>0$ and $\lambda_{\kappa}>0$, (depending on $\kappa)$ such that for all $\varepsilon \in\left(0, \varepsilon_{\kappa}\right), \lambda \in\left(0, \lambda_{\kappa}\right)$, for all set of parameter $\tilde{\eta}^{i}$, for $i=1, \ldots, m$, satisfying (40) and function $\tilde{\varphi}$ satisfying (23), there exists a

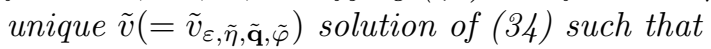

$$
\|\tilde{v}\|_{\mathcal{C}_{\nu}^{2, \alpha}\left(\bar{\Omega}^{*}(\mathbf{p} \cup \tilde{\mathbf{q}})\right)} \leq 2 c_{\kappa} r_{\varepsilon}^{2} .
$$

\section{The nonlinear Cauchy-data matching}

We gather the results of the previous sections, keeping the notations. Assume that $\tilde{\mathbf{q}}:=\left(\tilde{q}_{1}, \ldots, \tilde{q}_{m}\right) \in \Omega^{m}$ are given close to $\mathbf{q}:=\left(q_{1}, \ldots, q_{m}\right)$ and satisfy (30). Assume also that $\tau:=\left(\tau_{1}, \ldots, \tau_{K}\right) \in\left[\tau_{-}, \tau^{+}\right]^{K} \subset(0, \infty)^{K}$ are given (the values of $\tau_{-}$and $\tau^{+}$will be fixed shortly). First, we consider some set of boundary data $\varphi:=\left(\varphi^{1}, \ldots, \varphi^{K}\right) \in\left(\mathcal{C}^{2, \alpha}\left(S^{1}\right)\right)^{K}$ satisfying (20) and (21). According to the results of Propositions 4, 5, 6 and 7 and provided $\varepsilon \in\left(0, \varepsilon_{\kappa}\right)$ and $\lambda \in\left(0, \lambda_{\kappa}\right)$, we can find a solution of

$$
\Delta v+\lambda|\nabla v|^{2}+\varepsilon^{2} \prod_{i=1}^{N}\left|x-p_{i}\right|^{2 \beta_{i}} f(x)\left(e^{v}+e^{\gamma v}\right)=0
$$

which can be written as

$$
\begin{aligned}
v_{i n t}^{j_{i}}(x) & =v_{\varepsilon, \tau_{j_{i}}, \beta_{j_{i}}}\left(x-p_{j_{i}}\right)+h\left(x-p_{j_{i}}\right)-\log K_{j_{i}}^{1}(0) \\
& +H^{i}\left(\varphi^{j_{i}} ;\left(x-p_{j_{i}}\right) / r_{\varepsilon, \beta_{j_{i}}}\right)+\bar{v}_{\varepsilon, \tau_{j_{i}}, \varphi^{j_{i}}}\left(x-p_{j_{i}}\right),
\end{aligned}
$$

in $B_{r_{\varepsilon, \beta_{j_{i}}}}\left(p_{j_{i}}\right)$, where

$$
K_{j_{i}}^{1}(x)=\prod_{\ell=1, \ell \neq j_{i}}^{N}\left|x-p_{\ell}+p_{j_{i}}\right|^{2 \beta_{\ell}} f\left(x+p_{j_{i}}\right)
$$

and the function $v^{j_{i}}=\bar{v}_{\varepsilon, \tau_{j_{i}}, \varphi^{j_{i}}}$ satisfies

$$
\left\|v^{j_{i}}\right\|_{\mathcal{C}_{\mu}^{2, \alpha}\left(\mathbb{R}^{2}\right)} \leq 2 c_{\kappa} r_{\varepsilon, \beta_{j_{i}}} .
$$


Also a solution of $(48)_{\mid \beta_{i}=0}$ can be decomposed as

$v_{i n t}^{i}(x)=v_{\varepsilon, \tau_{i}}\left(x-\tilde{q}_{i}\right)+h\left(x-\tilde{q}_{i}\right)-\log K_{i}^{2}(0)+H^{i}\left(\varphi^{i} ;\left(x-\tilde{q}_{i}\right) / r_{\varepsilon}\right)+\bar{v}_{\varepsilon, \tau_{i}, \varphi^{i}}\left(x-\tilde{q}_{i}\right)$,

near $B_{r_{\varepsilon}}\left(\tilde{q}_{i}\right)$, where

$$
K_{i}^{2}(x)=\prod_{\ell=1}^{N}\left|x-p_{\ell}+\tilde{q}_{i}\right|^{2_{\ell}} f\left(x+\tilde{q}_{i}\right) .
$$

and the function $v^{i}=\bar{v}_{\varepsilon, \tau_{i}, \varphi^{i}}$ satisfies

$$
\left\|v^{i}\right\|_{\mathcal{C}_{\mu}^{2, \alpha}\left(\mathbb{R}^{2}\right)} \leq 2 c_{\kappa} r_{\varepsilon}^{2}
$$

Similarly, given some boundary data $\tilde{\varphi}:=\left(\tilde{\varphi}^{1}, \ldots, \tilde{\varphi}^{K}\right) \in\left(\mathcal{C}^{2, \alpha}\left(S^{1}\right)\right)^{K}$ satisfying (23), some parameters $\tilde{\eta}:=\left(\tilde{\eta}^{1}, \ldots, \tilde{\eta}^{K}\right) \in \mathbb{R}^{K}$ satisfying (36), (38) and (40), provided $\varepsilon \in\left(0, \varepsilon_{\kappa}\right)$, we use the result of Proposition 6 and Proposition 7 , to find a solution $v_{\text {ext }}$ of (48) which can be decomposed as

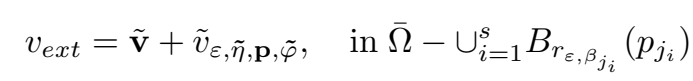

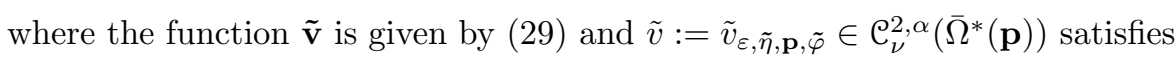

$$
\|\tilde{v}\|_{\mathcal{C}_{\nu}^{2, \alpha}\left(\bar{\Omega}^{*}(\mathbf{p})\right)} \leq 2 c_{\kappa} r_{\varepsilon, \bar{\beta}}
$$

and

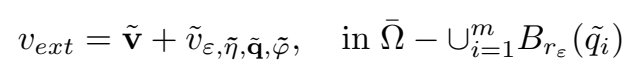

where the function $\tilde{\mathbf{v}}$ is given by $(29)$ and $\tilde{v}:=\tilde{v}_{\varepsilon, \tilde{\eta}, \tilde{\mathbf{q}}, \tilde{\varphi}} \in \mathcal{C}_{\nu}^{2, \alpha}\left(\bar{\Omega}^{*}(\mathbf{p} \cup \tilde{\mathbf{q}})\right)$ satisfies

$$
\|\tilde{v}\|_{\mathcal{C}_{\nu}^{2, \alpha}\left(\bar{\Omega}^{*}(\mathbf{p} \cup \tilde{\mathbf{q}})\right)} \leq 2 c_{\kappa} r_{\varepsilon}^{2} .
$$

It remains to determine the parameters and the functions in such a way that the function which is equal to $v_{i n t}^{j_{i}}$ in $B_{r_{\varepsilon, \beta_{j}}}\left(p_{j_{i}}\right)$ and is equal to $v_{e x t}$ in $\bar{\Omega}_{r_{\varepsilon, \beta_{j}}}(\mathbf{p})$ is smooth. Similarly it remains to determine the parameters and the functions is such a way that the function equal to $v_{i n t}^{i}$ in $B_{r_{\varepsilon}}\left(\tilde{q}_{i}\right)$ and which is equal to $v_{e x t}$ in $\left.\bar{\Omega}_{r_{\varepsilon}}(\mathbf{p} \cup \tilde{\mathbf{q}})\right)$ is smooth. This amounts to find the boundary data and the parameters so that

$$
v_{\text {int }}=v_{\text {ext }} \quad \text { and } \quad \partial_{r} v_{\text {int }}=\partial_{r} v_{\text {ext }},
$$

near $\partial B_{r_{\varepsilon, \beta_{j_{i}}}}\left(p_{j_{i}}\right)$ or near $\partial B_{r_{\varepsilon}}\left(\tilde{q}_{i}\right)$. Assuming we have already done so, this provides for each $\varepsilon$ small enough a function $v_{\varepsilon, \beta} \in \mathfrak{C}^{2, \alpha}$ (which is obtained 
by patching together the functions $v_{\text {int }}$ and the function $v_{\text {ext }}$ ) solution of our equation, the elliptic regularity theory implies that this solution is in fact smooth. This will complete the proof of our result since, as $\varepsilon$ and $\lambda$ tend to 0 , the sequence of solutions we have obtained satisfies the required properties, namely, away from the points $q_{i}$ the sequence $v_{\varepsilon, \beta}$ converges to some function in which appears the sum of $G\left(\cdot, q_{i}\right)$ in each case of our theorem.

Before we proceed, the following remarks are due. First, it will be convenient to observe that the functions $v_{\varepsilon, \tau_{j_{i}}, \beta_{j_{i}}}$ and $v_{\varepsilon, \tau_{i}}$ can be expanded as

$$
v_{\varepsilon, \tau_{j_{i}}, \beta_{j_{i}}}(x)=-2\left(1+\beta_{j_{i}}\right) \log \tau_{j_{i}}-4\left(1+\beta_{j_{i}}\right) \log |x|+\mathcal{O}\left(\frac{\varepsilon^{2} \tau_{j_{i}}^{-2\left(\beta_{j_{i}}+1\right)}}{|x|^{2\left(\beta_{j_{i}}+1\right)}}\right)
$$

near $\partial B_{r_{\varepsilon, \beta_{j}}}$ and

$$
v_{\varepsilon, \tau_{i}}(x)=-2 \log \tau_{i}-4 \log |x|+\mathcal{O}\left(\frac{\varepsilon^{2} \tau_{i}^{-2}}{|x|^{2}}\right)
$$

near $\partial B_{r_{\varepsilon}}$. The functions

$\sum_{\ell=1}^{K}\left(1+\beta_{j_{\ell}}+\tilde{\eta}^{j_{\ell}}\right) G\left(x, p_{j_{\ell}}\right) \quad$ and $\sum_{\ell=1}^{s}\left(1+\beta_{j_{\ell}}+\tilde{\eta}^{j_{\ell}}\right) G\left(x, p_{j_{\ell}}\right)+\sum_{\ell=1}^{m}\left(1+\tilde{\eta}^{\ell}\right) G\left(x, \tilde{q}_{\ell}\right)$

which appear in the expression of $v_{\text {ext }}$ can be expanded as

$$
\begin{aligned}
& \sum_{\ell=1}^{K}\left(1+\beta_{j_{\ell}}+\tilde{\eta}^{j_{\ell}}\right) G\left(x+p_{j_{i}}, p_{j_{\ell}}\right)=-4\left(1+\beta_{j_{i}}+\tilde{\eta}^{j_{i}}\right) \log |x|+ \\
& \left(1+\beta_{j_{i}}+\tilde{\eta}^{j_{i}}\right) H\left(x+p_{j_{i}}, p_{j_{i}}\right)+\sum_{\ell=1, \ell \neq i}^{K}\left(1+\beta_{j_{\ell}}+\tilde{\eta}^{j_{\ell}}\right) G\left(x+p_{j_{i}}, p_{j_{\ell}}\right)
\end{aligned}
$$

near $\partial B_{r_{\varepsilon, \beta_{j_{i}}}}$,

$$
\begin{aligned}
& \sum_{\ell=1}^{s}\left(1+\beta_{j_{\ell}}+\tilde{\eta}^{j^{\ell}}\right) G\left(x+p_{j_{i}}, p_{j_{\ell}}\right)+\sum_{\ell=1}^{m}\left(1+\tilde{\eta}^{\ell}\right) G\left(x+p_{j_{i}}, \tilde{q}_{\ell}\right) \\
= & -4\left(1+\beta_{j_{i}}+\tilde{\eta}^{j_{i}}\right) \log |x|+\left(1+\beta_{j_{i}}+\tilde{\eta}^{j_{i}}\right) H\left(x+p_{j_{i}}, p_{j_{i}}\right)+ \\
& \sum_{\ell=1, \ell \neq i}^{s}\left(1+\beta_{j_{\ell}}+\tilde{\eta}^{j_{\ell}}\right) G\left(x+p_{j_{i}}, p_{j_{\ell}}\right)+\sum_{\ell=1}^{m}\left(1+\tilde{\eta}^{\ell}\right) G\left(x+p_{j_{i}}, \tilde{q}_{\ell}\right)
\end{aligned}
$$


near $\partial B_{r_{\varepsilon, \beta_{j_{i}}}}$ and

$$
\begin{aligned}
& \sum_{\ell=1}^{s}\left(1+\beta_{j_{\ell}}+\tilde{\eta}^{j_{\ell}}\right) G\left(x+\tilde{q}_{i}, p_{j_{\ell}}\right)+\sum_{\ell=1}^{m}\left(1+\tilde{\eta}^{\ell}\right) G\left(x+\tilde{q}_{i}, \tilde{q}_{\ell}\right)= \\
& -4\left(1+\tilde{\eta}^{i}\right) \log |x|+\left(1+\tilde{\eta}^{i}\right) H\left(x+\tilde{q}_{i}, \tilde{q}_{i}\right)+\sum_{\ell=1, \ell \neq i}^{m}\left(1+\tilde{\eta}^{\ell}\right) G\left(x+\tilde{q}_{i}, \tilde{q}_{\ell}\right) \\
& +\sum_{\ell=1}^{s}\left(1+\beta_{j_{\ell}}+\tilde{\eta}^{j_{\ell}}\right) G\left(x+\tilde{q}_{i}, p_{j_{\ell}}\right)
\end{aligned}
$$

near $\partial B_{r_{\varepsilon}}$.

\subsection{End of the proof of part (a) of Theorem 1:}

In this case $S \cap \Lambda=S$, for $x$ near $\partial B_{r_{\varepsilon, \beta_{j}}}\left(p_{j_{i}}\right)$, we have

$$
\begin{aligned}
& \left(v_{i n t}-v_{e x t}\right)\left(x=-2\left(1+\beta_{j_{i}}\right) \log \tau_{j_{i}}+\log 8\left(1+\beta_{j_{i}}\right)^{2}+4 \tilde{\eta}^{j_{i}} \log \left|x-p_{j_{i}}\right|\right. \\
& +h\left(x-p_{j_{i}}\right)+H^{i}\left(\varphi^{j_{i}} ;\left(x-p_{j_{i}}\right) / r_{\varepsilon, \beta_{j_{i}}}\right)-H^{e}\left(\tilde{\varphi}^{j_{i}} ;\left(x-p_{j_{i}}\right) / r_{\varepsilon, \beta_{j_{i}}}\right) \\
& -\left(\left(1+\beta_{j_{i}}+\tilde{\eta}^{j_{i}}\right) H\left(x, p_{j_{i}}\right)+\log \left(\prod_{\ell=1, \ell \neq j_{i}}^{N}\left|p_{j_{i}}-p_{\ell}\right|^{2 \beta_{\ell}} f\left(p_{j_{i}}\right)\right)\right) \\
& -\sum_{\ell=1, \ell \neq i}^{s}\left(1+\beta_{j_{\ell}}+\tilde{\eta}^{j \ell}\right) G\left(x, p_{j_{\ell}}\right)+\mathcal{O}\left(\frac{\varepsilon^{2} \tau_{j_{i}}^{-2\left(\beta_{j_{i}}+1\right)}}{\left|x-p_{j_{i}}\right|^{2\left(\beta_{j_{i}}+1\right)}}\right) \\
& \left.=-2\left(1+\beta_{j_{i}}\right) \log \tau_{j_{i}}+\log 8\left(1+\beta_{j_{i}}\right)^{2}+4 \tilde{\eta}^{j_{i}} \log \left|x-p_{j_{i}}\right|+h\left(x-p_{j_{i}}\right)\right) \\
& +H^{i}\left(\varphi^{j_{i}} ;\left(x-p_{j_{i}}\right) / r_{\varepsilon, \beta_{j_{i}}}\right)-H^{e}\left(\tilde{\varphi}^{j_{i}} ;\left(x-p_{j_{i}}\right) / r_{\varepsilon, \beta_{j_{i}}}\right) \\
& -E_{j_{i}}\left(p_{j_{i}}, \mathbf{q}\right)+\mathcal{O}\left(\frac{\varepsilon^{2} \tau_{j_{i}}^{-2\left(\beta_{j_{i}}+1\right)}}{\left|x-p_{j_{i}}\right|^{2\left(\beta_{j_{i}}+1\right)}}\right)+\mathcal{O}\left(\left|x-p_{j_{i}}\right|\right) \\
& =-2\left(1+\beta_{j_{i}}\right) \log \tau_{j_{i}}+\log 8\left(1+\beta_{j_{i}}\right)^{2}+4 \tilde{\eta}^{j_{i}} \log r_{\varepsilon, \beta_{j_{i}}}-E_{j_{i}}\left(p_{j_{i}}, \mathbf{p}\right)+\mathcal{O}\left(r_{\varepsilon, \bar{\beta}}\right) \\
& =
\end{aligned}
$$


where $\mathbf{p}=\left(p_{j_{1}}, \cdots, p_{j_{s}}\right)$ and

$$
\begin{aligned}
& E_{j_{i}}(x, \mathbf{q})=\left(1+\beta_{j_{i}}\right) H\left(x, p_{j_{i}}\right)+\sum_{\ell=1, \ell \neq i}^{s}\left(1+\beta_{j_{\ell}}\right) G\left(x, p_{j_{\ell}}\right) \\
& +\log \left(\prod_{\ell=1, \ell \neq j_{i}}^{N}\left|x-p_{\ell}\right|^{2 \beta_{\ell}} f\left(p_{j_{i}}\right)\right) .
\end{aligned}
$$

Next, in (53), all functions are defined on $\partial B_{r_{\varepsilon, \beta_{i}}}\left(p_{j_{i}}\right)$ nevertheless, it will be convenient to solve instead of (53) the following set of equations

$$
\left(v_{\text {int }}-v_{\text {ext }}\right)\left(p_{j_{i}}+r_{\varepsilon, \bar{\beta}} \cdot\right)=0 \quad \text { and } \quad \partial_{r}\left(\left(v_{\text {int }}-v_{\text {ext }}\right)\left(p_{j_{i}}+r_{\varepsilon, \bar{\beta}} \cdot\right)\right)=0
$$

on $S^{1}$. Here all functions are considered as functions of $y \in S^{1}$ and we have simply used the change of variables $x=p_{j_{i}}+r_{\varepsilon, \bar{\beta}} y$ to parameterize $\partial B_{r_{\varepsilon, \beta_{j}}}\left(p_{j_{i}}\right)$. We decompose

$$
\varphi^{j_{i}}=\varphi_{0}^{j_{i}}+\varphi^{j_{i}, \perp} \quad \text { and } \quad \tilde{\varphi}^{j_{i}}=\tilde{\varphi}_{0}^{j_{i}}+\tilde{\varphi}^{j_{i}, \perp}
$$

where $\varphi_{0}^{j_{i}}, \tilde{\varphi}_{0}^{j_{i}}$ are constant functions on $S^{1}$, where $\varphi^{j_{i}, \perp}$ and $\tilde{\varphi}^{j_{i}, \perp}$ are $L^{2}\left(S^{1}\right)$ orthogonal to the constant functions.

Projecting the set of equations (53) over the constant functions, yields the system

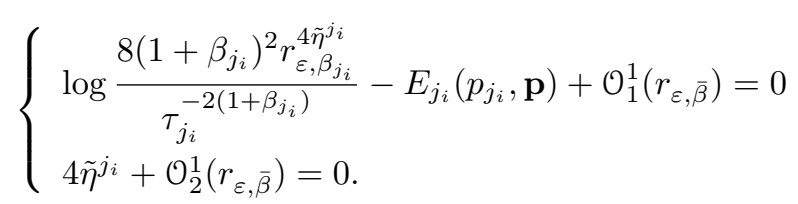

This system can be readily simplified into

$$
\frac{1}{\log r_{\varepsilon, \bar{\beta}}}\left(2\left(1+\beta_{j_{i}}\right) \log \tau_{j_{i}}-\log 8\left(1+\beta_{j_{i}}\right)^{2}+E_{j_{i}}\left(p_{j_{i}}, \mathbf{p}\right)\right)=\mathcal{O}_{3}^{1}\left(r_{\varepsilon, \bar{\beta}}\right)
$$

and

$$
\tilde{\eta}^{j_{i}}=\mathcal{O}_{4}^{1}\left(r_{\varepsilon, \bar{\beta}}\right),
$$

where the terms $\mathcal{O}_{k}^{1}\left(r_{\varepsilon, \bar{\beta}}\right)$ for $k=1 \cdots 4$ depend nonlinearly on $\tau_{\ell}, p_{\ell}, \varphi^{\ell}, \tilde{\varphi}^{\ell}$, but, they are bounded (in the appropriate norm) by a constant (independent of $\varepsilon$ and $\kappa$ ) time $r_{\varepsilon, \bar{\beta}}$. Let us comment briefly on how these equations are obtained. They simply come from (53) when expansions (54) and (56) are used, together with the expression of $H^{i}$ and $H^{e}$ given in Lemma 1 and Lemma 2, and also the estimates (49) and (51). 
We are now in position to define $\tau_{-}$and $\tau_{+}$since, according to the above, as $\varepsilon$ tends to 0 we expect that $\tau_{j_{i}}$ will converge to $\tau_{j_{i}}^{*}$ satisfying

$$
2\left(1+\beta_{j_{i}}\right) \log \tau_{j_{i}}^{*}-\log 8\left(1+\beta_{j_{i}}\right)^{2}=-E_{j_{i}}\left(p_{j_{i}}, \mathbf{p}\right)
$$

and hence it is enough to choose $\tau_{-}$and $\tau_{+}$in such a way that

$$
\log \frac{\tau_{-}^{2\left(1+\beta_{j_{i}}\right)}}{8\left(1+\beta_{j_{i}}\right)^{2}}<-\sup _{i} E_{j_{i}}\left(p_{j_{i}}, \mathbf{p}\right) \leq-\inf _{i} E_{j_{i}}\left(p_{j_{i}}, \mathbf{p}\right)<\log \frac{\tau_{+}^{2\left(1+\beta_{j_{i}}\right)}}{8\left(1+\beta_{j_{i}}\right)^{2}}
$$

Now, we consider the $L^{2}$-projection onto the space of functions which are $L^{2}$-orthogonal to the constant functions. This yields the system

$$
\left\{\begin{array}{l}
\varphi^{j_{i}, \perp}-\tilde{\varphi}^{j_{i}, \perp}+\mathcal{O}_{5}^{1}\left(r_{\varepsilon, \bar{\beta}}\right)=0 \\
\partial_{r}\left(H^{i, \perp}-H^{e, \perp}\right)+\mathcal{O}_{6}^{1}\left(r_{\varepsilon, \bar{\beta}}\right)=0
\end{array}\right.
$$

Thanks to the result of Lemma 3, this last system can be re-written as

$$
\varphi^{j_{i}, \perp}=\mathcal{O}_{7}^{1}\left(r_{\varepsilon, \bar{\beta}}\right)
$$

If we define the parameters $\mathbf{t}=\left(t^{j_{i}}\right) \in \mathbb{R}^{s}$ by

$t^{j_{i}}=\frac{1}{\log r_{\varepsilon, \bar{\beta}}}\left(2\left(1+\beta_{j_{i}}\right) \log \tau_{j_{i}}-\log 8\left(1+\beta_{j_{i}}\right)^{2}+E_{j_{i}}\left(p_{j_{i}}, \mathbf{p}\right)\right), \quad \forall 1 \leq i \leq s$

then the system we have to solve reads

$$
\left(\mathbf{t}, \tilde{\eta}, \varphi^{\perp}, \tilde{\varphi}^{\perp}\right)=\mathcal{O}_{8}^{1}\left(r_{\varepsilon, \bar{\beta}}\right)
$$

where as usual, the terms $\mathcal{O}_{k}^{1}\left(r_{\varepsilon, \bar{\beta}}\right), k=5 \cdots 8$ depend nonlinearly on $\mathbf{t}, \tilde{\eta}, \varphi^{\perp}$, $\tilde{\varphi}^{\perp}$ but they are bounded (in the appropriate norm) by a constant (independent of $\varepsilon$ and $\kappa$ ) time $r_{\varepsilon, \bar{\beta}}$.

The nonlinear mapping which appears on the right hand side of (63) is continuous and compact. In addition, this nonlinear mapping sends the ball of radius $\kappa r_{\varepsilon, \bar{\beta}}$ (for the natural product norm) into itself, provided $\kappa$ is fixed large enough. Applying Schauder's fixed point theorem in the ball of radius $\kappa r_{\varepsilon, \bar{\beta}}$ in the product space where the entries live yields the existence of a solution of equation (63) and this completes the proof of the part (a) of Theorem 1. 


\subsection{End of the proof of part (b) of Theorem 1:}

In this case $S \cap \Lambda=\emptyset$ thus for $x$ near $\partial B_{r_{\varepsilon}}$, we have

$$
\begin{aligned}
& \left(v_{i n t}-v_{\text {ext }}\right)(x) \\
& =-2 \log \tau_{i}+\log 8+4 \tilde{\eta}^{i} \log \left|x-\tilde{q}_{i}\right|+h\left(x-\tilde{q}_{i}\right)+H^{i}\left(\varphi^{i} ;\left(x-\tilde{q}_{i}\right) / r_{\varepsilon}\right) \\
& -H^{e}\left(\tilde{\varphi}^{i} ;\left(x-\tilde{q}_{i}\right) / r_{\varepsilon}\right)-\left(\left(1+\tilde{\eta}^{i}\right) H\left(x, \tilde{q}_{i}\right)+\sum_{\ell=1, \ell \neq i}^{m}\left(1+\tilde{\eta}^{\ell}\right) G\left(x, \tilde{q}_{\ell}\right)\right. \\
& \left.+\log \left(\prod_{\ell=1, \ell \neq i}^{N}\left|\tilde{q}_{i}-p_{\ell}\right|^{2 \beta_{\ell}} f\left(\tilde{q}_{i}\right)\right)\right)+\mathcal{O}\left(\frac{\varepsilon^{2} \tau_{i}^{-2}}{\left|x-\tilde{q}_{i}\right|^{2}}\right) \\
& =-2 \log \tau_{i}+\log 8+4 \tilde{\eta}^{i} \log |x|+h\left(x-\tilde{q}_{i}\right)+H^{i}\left(\varphi^{i} ;\left(x-\tilde{q}_{i}\right) / r_{\varepsilon}\right) \\
& -H^{e}\left(\tilde{\varphi}^{i} ;\left(x-\tilde{q}_{i}\right) / r_{\varepsilon}\right)-\left(\left(1+\tilde{\eta}^{i}\right) H\left(\tilde{q}_{i}, \tilde{q}_{i}\right)+\sum_{\ell=1, \ell \neq i}^{m}\left(1+\tilde{\eta}^{\ell}\right) G\left(\tilde{q}_{i}, \tilde{q}_{\ell}\right)\right. \\
& \left.+\log \left(\prod_{\ell=1, \ell \neq i}^{N}\left|\tilde{q}_{i}-p_{\ell}\right|^{2 \beta_{\ell}} f\left(\tilde{q}_{i}\right)\right)\right)+\mathcal{O}\left(\left|x-\tilde{q}_{i}\right|^{2}\right)+\mathcal{O}\left(\frac{\varepsilon^{2} \tau_{i}^{-2}}{\left|x-\tilde{q}_{i}\right|^{2}}\right)+\mathcal{O}\left(r_{\varepsilon}^{2}\right) \\
& =-2 \log \tau_{i}+\log 8+4 \tilde{\eta}^{i} \log r_{\varepsilon}-\mathcal{F}_{i}\left(\tilde{q}_{i}, \tilde{\mathbf{q}}\right)+\mathcal{O}\left(r_{\varepsilon}^{2}\right)
\end{aligned}
$$

where $\mathbf{q}=\left(q_{1}, \cdots, q_{m}\right)$ and

$$
\mathcal{F}_{i}(x, \tilde{\mathbf{q}})=H\left(x, \tilde{q}_{i}\right)+\sum_{\ell=1, \ell \neq i}^{m} G\left(x, \tilde{q}_{\ell}\right)+\log \left(\prod_{\ell=1, \ell \neq i}^{N}\left|x-p_{\ell}\right|^{2 \beta_{\ell}} f\left(\tilde{q}_{i}\right)\right) .
$$

Next, in (53), all functions are defined on $\partial B_{r_{\varepsilon}}\left(\tilde{q}_{i}\right)$, but it will be convenient to solve on $S^{1}$ the following equations

$$
\left(v_{i n t}-v_{e x t}\right)\left(\tilde{q}_{i}+r_{\varepsilon} \cdot\right)=0 \quad \text { and } \quad \partial_{r}\left(\left(v_{i n t}-v_{e x t}\right)\left(\tilde{q}_{i}+r_{\varepsilon} \cdot\right)\right)=0 .
$$

We decompose

$$
\varphi^{i}=\varphi_{0}^{i}+\varphi_{1}^{i}+\varphi^{i, \perp} \quad \text { and } \quad \tilde{\varphi}^{i}=\tilde{\varphi}_{0}^{i}+\tilde{\varphi}_{1}^{i}+\tilde{\varphi}^{i, \perp}
$$

where $\varphi_{0}^{i}, \tilde{\varphi}_{0}^{i} \in \mathbb{E}_{0}=\mathbb{R}$ are constant functions on $S^{1}, \varphi_{1}^{i}, \tilde{\varphi}_{1}^{i}$ belong to $\mathbb{E}_{1}=$ $\operatorname{ker}\left(\Delta_{S^{1}}+1\right)=\operatorname{Span}\left\{e_{1}, e_{2}\right\}$ and where $\varphi^{i, \perp}, \tilde{\varphi}^{i, \perp}$ are $L^{2}\left(S^{1}\right)$ orthogonal to $\mathbb{E}_{0}$ and $\mathbb{E}_{1}$. 
Projecting the equations $(65)$ over $\mathbb{E}_{0}$ will yield the system

$$
\left\{\begin{aligned}
-2 \log \tau_{i}+\log 8+4 \tilde{\eta}^{i} \log r_{\varepsilon}-\mathcal{F}_{i}\left(\tilde{q}_{i}, \tilde{\mathbf{q}}\right)+\mathcal{O}_{1}^{2}\left(r_{\varepsilon}^{2}\right) & =0 \\
4 \tilde{\eta}^{i}+\mathcal{O}_{2}^{2}\left(r_{\varepsilon}^{2}\right) & =0 .
\end{aligned}\right.
$$

Let us comment briefly on how these equations are obtained. They simply come from (65) when expansions (55) and (58) are used, together with the expression of $H^{i}$ and $H^{e}$ given in Lemma 1 and Lemma 2, and also the estimates (50) and (52). The system (66) can be readily simplified into

$$
\frac{1}{\log r_{\varepsilon}}\left[2 \log \tau_{i}-\log 8+\mathcal{F}_{i}\left(\tilde{q}_{i}, \tilde{\mathbf{q}}\right)\right]=\mathcal{O}_{3}^{2}\left(r_{\varepsilon}^{2}\right), \quad \text { and } \quad \tilde{\eta}^{i}=\mathcal{O}_{4}^{2}\left(r_{\varepsilon}^{2}\right)
$$

We are now in a position to define $\tau_{-}$and $\tau^{+}$since, according to the above, as $\varepsilon$ tends to 0 we expect that $\tilde{q}_{i}$ will converge to $q_{i}$ and that $\tau_{i}$ will converge to $\tau_{i}^{*}$ satisfying

$$
2 \log \tau_{i}^{*}-\log 8=-\mathcal{F}_{i}\left(q_{i}, \mathbf{q}\right)
$$

and hence it is enough to choose $\tau_{-}$and $\tau^{+}$in such a way that

$$
2 \log \left(\tau_{-}\right)-\log 8<-\sup _{i} \mathcal{F}_{i}\left(q_{i}, \mathbf{q}\right) \leq-\inf _{i} \mathcal{F}_{i}\left(q_{i}, \mathbf{q}\right)<2 \log \left(\tau^{+}\right)-\log 8
$$

We now consider the $L^{2}$-projection of $(65)$ over $\mathbb{E}_{1}$. Given a smooth function $f$ defined in $\Omega$, we identify its gradient $\nabla f=\left(\partial_{x_{1}} f, \partial_{x_{2}} f\right)$ with the element of $\mathbb{E}_{1}$

$$
\bar{\nabla} f=\sum_{i=1}^{2} \partial_{x_{i}} f e_{i}
$$

With these notations in mind, we obtain the equations

$$
\bar{\nabla} \mathcal{F}_{i}\left(\tilde{q}_{i}, \tilde{\mathbf{q}}\right)=\mathcal{O}\left(r_{\varepsilon}^{2}\right) \text { and } \quad \varphi_{1}^{i}=\mathcal{O}_{5}^{2}\left(r_{\varepsilon}^{2}\right) .
$$

Finally, we consider the $L^{2}$-projection onto $L^{2}\left(S^{1}\right)^{\perp}$. This yields the system

$$
\left\{\begin{aligned}
\varphi^{i, \perp}-\tilde{\varphi}^{i, \perp}+\mathcal{O}_{6}^{2}\left(r_{\varepsilon}^{2}\right) & =0 \\
\partial_{r}\left(H^{i, \perp}-H^{e, \perp}\right)+\mathcal{O}_{7}^{2}\left(r_{\varepsilon}^{2}\right) & =0
\end{aligned}\right.
$$

Thanks to the result of Lemma 3, this last system can be re-written as

$$
\varphi^{i, \perp}=\mathcal{O}_{8}^{2}\left(r_{\varepsilon}^{2}\right) \quad \text { and } \quad \tilde{\varphi}^{i, \perp}=\mathcal{O}_{9}^{2}\left(r_{\varepsilon}^{2}\right) .
$$

If we define the parameters $\mathbf{t}=\left(t_{i}\right) \in \mathbb{R}^{m}$ by

$$
t_{i}=\frac{1}{\log r_{\varepsilon}}\left[2 \log \tau_{i}-\log 8+\mathcal{F}_{i}\left(\tilde{q}_{i}, \tilde{q}\right)\right], \quad \forall 1 \leq i \leq m .
$$


Then the system we have to solve reads

$$
\left(\mathbf{t}, \tilde{\eta}, \bar{\nabla} \mathcal{F}(\tilde{\mathbf{q}}), \varphi^{\perp}, \tilde{\varphi}^{\perp}\right)=\mathcal{O}_{10}^{2}\left(r_{\varepsilon}^{2}\right),
$$

where as usual, the terms $\mathcal{O}_{k}^{2}\left(r_{\varepsilon}^{2}\right), k=1 \cdots 10$ depend nonlinearly on all the variables on the left side, but they are bounded (in the appropriate norm) by a constant (independent of $\varepsilon$ and $\kappa$ ) time $r_{\varepsilon}^{2}$, provided $\varepsilon \in\left(0, \varepsilon_{\kappa}\right)$.

We claim, provided that the degree of the mapping

$$
\bar{\nabla} \mathcal{F}: \tilde{\mathbf{q}} \longmapsto\left(\bar{\nabla} \mathcal{F}_{1}\left(\tilde{q}_{1} ; \tilde{\mathbf{q}}\right), \ldots, \bar{\nabla} \mathcal{F}_{m}\left(\tilde{q}_{m} ; \tilde{\mathbf{q}}\right)\right),
$$

from a neighborhood of $\mathbf{q} \in \Omega^{m}$ to a neighborhood of 0 in $\mathbb{E}_{1}^{m}$ is equal to 1 , this nonlinear system can be solved by using Schauder's fixed point theorem in the ball of radius $\kappa r_{\varepsilon}^{2}$ in the product space where the entries live, namely

$$
\mathbf{t}, \eta \in \mathbb{R}^{m} ; \quad r_{\varepsilon}(\tilde{\mathbf{q}}-\mathbf{q}) \in\left(\mathbb{R}^{2}\right)^{m}
$$

and

$$
\varphi^{\perp}, \tilde{\varphi}^{\perp} \in\left(\mathcal{C}^{2, \alpha}\left(S^{1}\right)^{\perp}\right)^{m} .
$$

As before, the nonlinear mapping which appears on the right hand side of (69) is continuous and compact. In addition, this nonlinear mapping sends the ball of radius $\kappa r_{\varepsilon}^{2}$ (for the natural product norm) into itself, provided $\kappa$ is fixed large enough. Applying Schauder's fixed Theorem in the ball of radius $\kappa r_{\varepsilon}^{2}$ in the product space where the entries live the existence of a solution of equation (69). This ends the proof of the part (b) of Theorem 1.

\subsection{End of the proof of part (c) of Theorem 1:}

In this case $S \cap \Lambda=\left\{p_{j_{1}}, \cdots, p_{j_{s}}\right\}$ and $S \backslash \Lambda=\left\{q_{1}, \cdots, q_{m}\right\}$ with $s+m=K$. The remaining of the analysis of the previous subsections remains essentially unchanged, we simply combine the two previous constructions by gluing the limits functions and at once the definition of $E_{i}$ is modified into

$$
E_{i}(x,(\mathbf{p}, \tilde{\mathbf{q}}))=\mathcal{F}_{i}(x, \tilde{\mathbf{q}})+\mathcal{G}(x, \mathbf{p})
$$

where

$$
\mathcal{F}_{i}(x, \tilde{\mathbf{q}})=H\left(x, \tilde{q}_{i}\right)+\sum_{\ell=1, \ell \neq i}^{m} G\left(x, \tilde{q}_{\ell}\right)+\log \left(\prod_{\ell=1, \ell \neq i}^{N}\left|x-p_{\ell}\right|^{2 \beta_{\ell}} f\left(\tilde{q}_{i}\right)\right)
$$

and

$$
\mathcal{G}(x, \mathbf{p})=\sum_{\ell=1}^{s}\left(1+\beta_{j_{\ell}}\right) G\left(x, p_{j_{\ell}}\right) .
$$




\section{Acknowledgments}

This project was supported by King Saud University, Deanship of Scientific Research, College of Science Research Center.

\section{References}

[1] I. Abid, S. Baraket, T. Ouni and N. Trabelsi, Singular limits solutions for 2-dimensional elliptic problem with exponentially dominated nonlinearity with nonlinear gradient term, Boundary Value Problems (2011), 2011:10.

[2] S. Baraket, I. Bazarbacha and N. Trabelsi Construction of singular limits for four-dimensional elliptic problems with exponentially dominated nonlinearity, Bull. Sci. math. 131 (2007) 670-685.

[3] S. Baraket, I. Ben Omrane and T. Ouni, Singular limits for 2-dimensional elliptic problem involving exponential with nonlinear gradient term. Nonlinear Differ. Equ. Appl. 18 (2011), 59-78.

[4] S. Baraket, I. Ben. Omrane, T. Ouni and N. Trabelsi, Singular limits solutions for 2-dimensional elliptic problem with exponentially dominated nonlinearity and singular data, Communications in Contemporary Mathematics Vol. 13, No. 4 (2011) 697-725.

[5] S. Baraket, M. Dammak, T. Ouni and F. Pacard, Singular limits for a 4-dimensional semilinear elliptic problem with exponential nonlinearity, Ann. I. H. Poincaré - AN 24 (2007) 875-895.

[6] S. Baraket and F. Pacard, Construction of singular limits for a semilinear elliptic equation in dimension 2, Calc. Var. Partial Differential Equations 6 (1998), 1-38.

[7] S. Baraket and D. Ye, Singular limit solutions for two-dimensional elliptic problems with exponentionally dominated nonlinearity, Chinese Ann. Math. Ser. B, 22 (2001), 287-296.

[8] D. Bartolucci, C.C. Chen, C. S. Lin, G. Tarantello, Profile of blow-up solutions to mean field equations with singular data. Comm. Partial Differential Equations 29 (2004), no. 7-8, 1241-1265.

[9] S.Y.A. Chang, On a fourth order differential operator-the Paneitz operator in conformal geometry, Proceedings conference for the $70^{\text {th }}$ birthday of A.P.Calderon, 4, (2000), 155-165. 
[10] C. C. Chen, C. S. Lin, Mean field equations of liouville type with singular data: sharper estimates. Discrete contin. Dyn. Syst. 28(2010), no.43, 1237-1272.

[11] M. Dammak and T. Ouni, Singular limits for 4-dimensional semilinear elliptic problem with exponential nonlinearity adding a singular source term given by Dirac masses, Differential and Integral Equations 11-12 (2008), 1019-1036.

[12] M. Del Pino, M. Kowalczyk and M. Musso, Singular limits in Liouville type equations, Calc. Var. Partial Differential Equations 24 (2005), 47-81.

[13] P. Esposito, Blow up solutions for a Liouville equation with singular data, , SIAM J. Math. Anal. 36 (2005), 1310-1345.

[14] P. Esposito, M. Grossi and A. Pistoia, On the existence of Blowing-up solutions for a mean field equation,Ann. I. H. Poincaré - AN 22 (2005) 227-257.

[15] J. Liouville, Sur l'équation aux différences partielles $\partial^{2} \log \frac{\lambda}{\partial u \partial v} \pm \frac{\lambda}{2 a^{2}}=0$, J. Math. 18 (1853), 17-72.

[16] R. Mazzeo, Elliptic theory of edge operators I, Comm. Partial Differential Equations 16, 10 (1991) 1616-1664.

[17] R. Melrose, The Atiyah-Patodi-Singer Index Theorem, Res. Notes Math; vol. 4, 1993.

[18] K. Nangasaki and T. Suzuki, Asymptotic analysis for two-dimensional elliptic eigenvalue problems with exponentially dominated nonlinearities, Asymptotic Anal. 3 (1990), 173-188.

[19] F. Pacard and T. Rivière, Linear and Nonlinear Aspects of Vortices: the Ginzburg Landau Model, Progress in Nonlinear Differential Equations, vol. 39, Birkäuser, 2000.

[20] T. Suzuki, Tow dimensional Emden-Fowler equation with exponential nonlinearity, Nonlinear Diffusion Equations and their equilibrium states 3, Birkäuser (1992) 493-512.

[21] G. Tarantello, On Chern-Simons Theory Nonlinear PDE's and physical modeling: superfluidity, superconductivity and reactive flows, H. Berestycki ed. Kluver Academic Publisher. 
[22] J. Wei, D. Ye and F. Zhou, Bubbling solutions for an anisotropic EmdenFowler equation, Calc. Var. Partial Differential Equations 28 (2) (2007) 217-247.

[23] H. C. Wente, Counter example to a conjecture of H. Hopf, Pacific J.Math.121 (1986), 193-243.

[24] V. H. Weston, On the asymptotique solution of a partial differential equation with exponential nonlinearity, SIAM J. Math. 9 (1978), 1030-1053.

[25] D. Ye and F. Zhou, A generalized two dimensional Emden-Fowler equation with exponential nonlinearity, Calc. Var. Partial Differential Equations 13 (2001), 141-158.

[26] C. Zhao, Singular limits in a Liouville-type equation with singular sources, Houston J. Math. 34 (2008), no. 2, 601-621.

[27] C. Zhao, Blowing-up solutions to an anisotropic Emden-Fowler equation with a singular source, J. Math. Anal. Appl. 342 (2008) 398-422.

Sami BARAKET,

Department of Mathematics,

College of Science, King Saud University,

P.O. Box 2455, Riyadh 11451, Saudi Arabia.

Email: sbaraket@ksu.edu.sa

Imen BAZARBACHA,

Département de Mathématiques,

Faculté des Sciences de Tunis,

Campus Universitaire 2092 Tunis, Tunisie.

Email: Imen.Bacha@yahoo.fr

Saber KHARRATI,

Département de Mathématiques,

Faculté des Sciences de Tunis,

Campus Universitaire 2092 Tunis, Tunisie.

Email: kharrati.saber@gmail.com

Taieb OUNI,

Département de Mathématiques,

Faculté des Sciences de Tunis,

Campus Universitaire 2092 Tunis, Tunisie.

Email: Taieb.Ouni@fst.rnu.tn 